\title{
Non-combability of Hilbert modular groups
}

\author{
TOSHIAKI HATTORI
}

\section{Introduction.}

In the recent study of finitely generated infinite groups, important classes of groups have been defined and introduced by J.W. Cannon, M. Gromov, D.B.A. Epstein, D.F. Holt, S.V.F. Levy, M.S. Patterson, and W.P. Thurston: hyperbolic groups ([12]), automatic groups, combable groups ([5]), etc.. It has turned out that many well known classes of finitely generated groups belong (or do not belong) to them ([5, 8-12]). But so far not much seems to be known for arithmetic groups except the cocompact lattices in R-rank 1 semisimple Lie groups (which are Gromov hyperbolic). First we recall the known results:

(1) The group $S L(n, \mathbf{Z})$ is hyperbolic if $n=2$ [11], and is not combable if $n \geqq 3([5])$.

(2) For the ring $\mathcal{O}$ of algebraic integers of each imaginary quadratic number field, $S L(2, \mathcal{O})$ is automatic, because it is the fundamental group of a noncompact complete hyperbolic 3-manifold of finite volume, so is geometrically finite ([5]).

(3) Let $d \geqq 2$ be a square-free integer and $\mathcal{O}=\mathcal{O}(\sqrt{d})$ the ring of algebraic integers in the real quadratic number field $\mathbf{Q}(\sqrt{d})$. Then the group $S L(2, \mathcal{O})$ is not biautomatic ([10]).

In this article, we show the following.

Theorem. Let $\mathbf{k}(\neq \mathbf{Q})$ be a totally real algebraic number field and $\mathcal{O}_{\mathbf{k}}$ the ring of algebraic integers of $\mathbf{k}$. Then the group $S L\left(n, \mathcal{O}_{\mathbf{k}}\right)$ is not combable (therefore, not automatic) for $n \geqq 2$.

Remark. (1)There are several distinct notions of combing (see $[1,5,8]$ ). We claim in the above theorem that $S L\left(n, \mathcal{O}_{\mathbf{k}}\right)$ is not combable in the sense of Epstein et al. ([5]).

(2) The case $n=[\mathbf{k}: \mathbf{Q}]=2$ is one of the questions raised in [10]. And this gives the first example of arithmetic subgroups of Q-rank 1 algebraic 
groups which are not combable. It seems that the description in $[13, \S 3 . \mathrm{H}]$ is related to this case.

One of the central ideas in geometric group theory is that if a group acts isometrically, properly discontinuously, and cocompactly on a geodesic proper metric space, the geometry of the space affects the combinatorial nature of the group. The most natural space on which the arithmetic group $S L\left(n, \mathcal{O}_{\mathrm{k}}\right)$ acts is probably the corresponding symmetric space $X$. Unfortunately the quotient space is not compact in this case. But we can consider the submanifold $X^{\prime}$ obtained by removing the portions corresponding to the ends of the quotient. In fact, Epstein and Thurston ([5]) investigated such a submanifold of the symmetric space $S O(n) \backslash S L(n, \mathbf{R})$ in the case $S L(n, \mathbf{Z})$ $(n \geqq 3)$. We clarify the geometric meaning of their argument and apply the method to wider classes of arithmetic groups.

They showed the following general result ([5, Theorem 10.3.5]): If a combable group acts on a $k$-connected Riemannian manifold, there exists a positive constant $C$ with the following property. Let $z$ be a lipschitz $k$-cycle in the manifold. Then there exists a lipschitz $(k+1)$-chain $u$, such that $\partial u=z$ and that $\operatorname{mass}_{k+1}(u) \leqq C \cdot \operatorname{diam}(z) \operatorname{mass}_{k}(z)$, where $\operatorname{mass}_{i}$ is the $i$-dimensional volume for $i=k, k+1$ and $\operatorname{diam}(z)$ is the diameter of $z$.

Thus the problem turns to finding a $k$-cycle $z_{m}$ for each positive integer $m$ such that the volume of $z_{m}$ grows much faster than the infimum of the volumes of $(k+1)$-chains filling $z_{m}$ as $m$ goes to infinity. And this is a matter of geometry of nonpositively curved manifolds.

In this part, the most important points of their argument are the following:

(1) The cycles $z_{m}$ are constructed in a horosphere with respect to some suitable geodesic.

(2) In order to estimate the infimum of the volumes of chains filling $z_{m}$, they used a bounded closed differential form on the submanifold.

First we explain the former briefly. Horospheres in higher rank symmetric spaces have interesting metrical property while every horosphere (equipped with the induced Riemannian metric) in manifolds of constant negative sectional curvature is just the Euclidean space. For example, the typical horosphere in the Riemannian direct product $\mathcal{H} \times \mathcal{H}$ of the upper half planes, where each factor $\mathcal{H}$ has constant sectional curvature $-1 / 2$, is isometric to $\mathbf{R}^{3}$ equipped with the metric $d s^{2}=e^{z} d x^{2}+e^{-z} d y^{2}+d z^{2}$. Let $S_{0}$ be a regular square in the $x y$-plane such that one pair of its opposite edges is parallel to the $x$-axis, the other pair is parallel to the $y$-axis, and all the edges have length $2 \sqrt{2} e^{2 m}$. If we push upward (resp. downward) $S_{0}$ along the $z$-axis till the $z$-coordinates change to $4 m$ (resp. $-4 m$ ), then the 
length of the edges parallel to the $y$-axis (resp. the $x$-axis) of the resulting rectangle $S_{+}$(resp. $S_{-}$) is equal to $2 \sqrt{2}$ (see Figure 2 in $\S 3$ ). By using this property, we can construct 2-chain whose area is much larger than the length of its boundary. The situation is the same for $S O(n) \backslash S L(n, \mathbf{R})$. In case of other symmetric spaces, if we take a geodesic $\gamma$ in some suitable direction, we can describe the metric of the horospheres with respect to $\gamma$ by using the Langlands decomposition of the (standard) maximal parabolic Q-subgroup (Lemma 2.1).

Concerning the latter, we use the pullback of the volume form on the unipotent part of the Langlands decomposition (see $\S 4$ ). Since this form $\Omega$ is not bounded on the whole space $X$, we need to know the structure of $X^{\prime}$ near its boundary to some extent. In the case $n \geqq 3$, the structure of the end of the quotient is complicated, and we cannot know $X^{\prime}$ explicitly. But we can compute the norm of $\Omega$ explicitly on each horosphere (Lemma 4.2). So it suffices for our aim to prove that the submanifold $X^{\prime}$ is contained in the complement of a horoball with respect to $\gamma$. We show this by estimating the supremum of the injectivity radii on each horosphere (Lemma 5.7).

The organization of this paper is as follows. In $\S 1$, we take a finite index, normal subgroup $\Gamma^{\prime}$ of $\Gamma=S L\left(n, \mathcal{O}_{\mathrm{k}}\right)$ and find a submanifold $X^{\prime}$ of the corresponding symmetric space $X$ on which $\Gamma$ acts cocompactly. In $\S 2$, we take a geodesic $\gamma$ and describe the horospheres of $X$ with respect to $\gamma$ in terms of parabolic subgroups. We construct (preliminary) cycles $z_{m}$ in $\S 3$ (, which we replace by new cycles $\mathcal{Z}_{m}$ in $\S 5$ ). In $\S 4$, we define a closed form $\Omega$ on $X$ and compute its norm. In $\S 5$, we complete the proof of Theorem by finding a lower bound of the volumes of chains filling $\mathcal{Z}_{m}$.

\section{Notation.}

(1) We denote by $\mathbf{C}, \mathbf{R}, \mathbf{Q}, \mathbf{Z}, \mathbf{R}^{+}, \mathbf{C}^{*}$ the set of the complex numbers, the real numbers, the rational numbers, the (rational) integers, the positive real numbers, the nonzero complex numbers, respectively.

(2) For any algebraic group $H$ defined over $Q$, we denote by $H_{R}, H_{Q}, H_{Z}$ the group of real points, rational points, integral points, respectively, of $\mathbf{H}$. And for any algebraic group $\mathbf{H}$ (resp. Lie group $L$ ), we denote by $\mathbf{H}^{0}$ (resp. $L^{0}$ ) the identity component of $\mathbf{H}$ (resp. $L$ ) in the Zariski topology (resp. in its topology as a Lie group). 


\section{Preliminaries.}

Let $\mathbf{k}$ be a totally real algebraic number field and $\mathcal{O}_{\mathbf{k}}$ the ring of algebraic integers of $\mathbf{k}$. Let $l=[\mathbf{k}: \mathbf{Q}] \geqq 2$ be the degree of $\mathbf{k}$ over $\mathbf{Q}$. Then the group $S L\left(n, \mathcal{O}_{\mathbf{k}}\right)$ acts isometrically on the Riemannian product of $l$ copies of the symmetric space $S O(n) \backslash S L(n, \mathbf{R})$. In this section, for the argument of the remainder sections, we regard $S L\left(n, \mathcal{O}_{\mathbf{k}}\right)$ as the group $\Gamma=\mathbf{G}_{\mathbf{z}}$ of integral points of a certain algebraic $\mathbf{Q}$-group $\mathbf{G}$. The general reference of this section is [18, Chap.6].

We recall that $\mathbf{k}=\mathbf{Q}(\theta)$ for some algebraic number $\theta$. Let $\theta_{1}, \ldots, \theta_{l}$ $\left(\theta_{1}=\theta\right)$ be the $l$ distinct roots of the minimum polynomial of $\theta$ over $\mathbf{Q}$. For each $i=1, \ldots, l$, we denote by $\phi_{i}$ the field embedding $\mathbf{k} \longrightarrow \mathbf{C}$ such that $\phi_{i}(\theta)=\theta_{i}$. We remark that $\phi_{1}=i d$. and that each $\phi_{i}$ can be extended to a field automorphism $\mathbf{C} \longrightarrow \mathbf{C}$. Let $\left\{\alpha_{1}, \ldots, \alpha_{l}\right\}$ be an integral basis of $\mathbf{k}$ : i.e. $\mathcal{O}_{\mathbf{k}}=\mathbf{Z} \alpha_{1}+\cdots+\mathbf{Z} \alpha_{l}, \mathbf{k}=\mathbf{Q} \alpha_{1}+\cdots+\mathbf{Q} \alpha_{l}$. For each $i, j \in$ $\{1, \ldots, l\}$, let $\alpha_{i} \alpha_{j}=\sum_{k=1}^{l} b_{i j}^{k} \alpha_{k} ; b_{i j}^{k} \in \mathbf{Z}$. We denote by $I_{n}$ the $n \times n$-matrix $\operatorname{diag}(1, \ldots, 1)$ and put

$$
\begin{gathered}
\mathbf{S}=\left(\phi_{j}\left(\alpha_{i}\right) I_{n}\right)=\left(\begin{array}{cccc}
\phi_{1}\left(\alpha_{1}\right) I_{n} & \phi_{2}\left(\alpha_{1}\right) I_{n} & \cdots & \phi_{l}\left(\alpha_{1}\right) I_{n} \\
\phi_{1}\left(\alpha_{2}\right) I_{n} & \phi_{2}\left(\alpha_{2}\right) I_{n} & \cdots & \phi_{l}\left(\alpha_{2}\right) I_{n} \\
\vdots & \vdots & & \vdots \\
\phi_{1}\left(\alpha_{l}\right) I_{n} & \phi_{2}\left(\alpha_{l}\right) I_{n} & \cdots & \phi_{l}\left(\alpha_{l}\right) I_{n}
\end{array}\right) \\
\mathbf{B}_{k}=\left(b_{k i}^{j} I_{n}\right) ; k=1, \ldots l .
\end{gathered}
$$

For each $k$ and $h=\left(h_{i j}\right) \in S L(n, \mathbf{C})$, we denote by $\phi_{k}(h)$ the element $\left(\phi_{k}\left(h_{i j}\right)\right)$ of $S L(n, \mathbf{C})$. We define a faithful representation $\iota: S L(n, \mathbf{C}) \longrightarrow$ $G L(n l, \mathbf{C})$ by

$$
\iota(h)=\operatorname{diag}\left(\phi_{1}(h), \ldots, \phi_{l}(h)\right) \quad \text { for } h \in S L(n, \mathbf{C}) .
$$

We also define another faithful representation $\rho: S L(n l, \mathbf{C}) \longrightarrow G L(n l, \mathbf{C})$ by $\rho(g)=\mathbf{S}^{-1} g \mathbf{S}$ for $g \in S L(n l, \mathbf{C})$. Let $\mathfrak{g l}(n l, \mathbf{C})$ be the set of $(n l) \times(n l)$ complex matrices. For

$$
Z=\left(Z_{i j}\right)=\left(\begin{array}{cccc}
Z_{11} & Z_{12} & \cdots & Z_{1 l} \\
Z_{21} & Z_{22} & \cdots & Z_{2 l} \\
\vdots & \vdots & & \vdots \\
Z_{l 1} & Z_{l 2} & \cdots & Z_{l l}
\end{array}\right) \in \mathfrak{g l}(n l, \mathbf{C})
$$


where each $Z_{i j}$ is an $n \times n$-matrix, we consider the following system of linear equations:

$$
\left(\begin{array}{llll}
Z_{i 1} & Z_{i 2} & \ldots & Z_{i l}
\end{array}\right)=\left(\begin{array}{llll}
Z_{11} & Z_{12} & \ldots & Z_{1 l}
\end{array}\right) \mathbf{B}_{1}^{-1} \mathbf{B}_{i} ; i=2, \ldots, l .
$$

We also consider the following equations:

$$
\operatorname{det}\left(p_{r} \circ \rho(Z)\right)=1 ; r=1, \ldots, l,
$$

where $p_{r}: \mathfrak{g l}(n l, \mathbf{C}) \longrightarrow \mathfrak{g l}(n, \mathbf{C})$ is the map defined by $p_{r}(Y)=Y_{r r}$ for $Y=\left(Y_{i j}\right) \in \mathfrak{g l}(n l, \mathbf{C})$. Let $\mathbf{G}(\subset S L(n l, \mathbf{C}))$ be the algebraic Q-group defined by the equations (1.1) and (1.2). Then $\mathbf{G}$ is "the group obtained from $S L(n, \mathbf{C})$ by the restriction of scalars from $\mathbf{k}$ to $\mathbf{Q}$ " in the sense of $[19$, Chap.I]. And we have $\mathbf{G}=\left(\rho^{-1} \circ \iota\right)(S L(n, \mathbf{C})), \mathbf{G}_{\mathbf{Z}}=\left(\rho^{-1} \circ \iota\right)\left(S L\left(n, \mathcal{O}_{\mathbf{k}}\right)\right)$. We put $\Gamma=\mathbf{G}_{\mathbf{z}}$ and identify $\Gamma$ with $S L\left(n, \mathcal{O}_{\mathbf{k}}\right)$. We also have

$$
\mathbf{G}_{\mathbf{R}}=\rho^{-1}\left(\left\{\operatorname{diag}\left(B_{1}, \ldots, B_{l}\right) \mid B_{1}, \ldots, B_{l} \in S L(n, \mathbf{R})\right\}\right) .
$$

The group $\mathbf{G}_{\mathbf{R}}$ is isomorphic to the direct product of $l$ copies of $S L(n, \mathbf{R})$. Let

$$
K=\rho^{-1}\left(\left\{\operatorname{diag}\left(K_{1}, \ldots, K_{l}\right) \mid K_{1}, \ldots, K_{l} \in S O(n)\right\}\right) .
$$

Then $K$ is a maximal compact subgroup of $\mathbf{G}_{\mathbf{R}}$ and is isomorphic to the direct product of $l$ copies of $S O(n)$.

We put $X=K \backslash \mathbf{G}_{\mathbf{R}}$, and give it the standard metric induced from the Killing form of the Lie algebra of $\mathbf{G}_{\mathbf{R}}$. Then $X$ is isometric to the Riemannian product of $l$ copies of $S O(n) \backslash S L(n, \mathbf{R})$ equipped with the standard metric. (Notice that $S O(2) \backslash S L(2, \mathrm{R})$ has constant sectional curvature $-1 / 2$ when we give it the standard metric induced from the Killing form.) The discrete group $\Gamma$ acts isometrically on the symmetric space $X$ from the right. We take a torsion-free, normal subgroup $\Gamma^{\prime}$ of $\Gamma$ of finite index.

By Raghunathan's construction ([17]), there exists a $\Gamma$-invariant smooth function $\tilde{f}: X \longrightarrow \mathbf{R}$ such that the induced smooth function $f$ on the quotient manifold $X / \Gamma^{\prime}$ satisfies the following conditions: (1) $f$ is proper, (2) $f$ has no critical points outside some compact subset, (3) the image of $f$ is $\left[t_{0}, \infty\right)$ for some positive number $t_{0}$.

Then, for sufficiently large $t>0, f^{-1}\left(\left[t_{0}, t\right]\right)$ is a deformation retract of $X / \Gamma^{\prime}$. If we put $X_{t}=\widetilde{f}^{-1}\left(\left[t_{0}, t\right]\right), X_{t}$ is a submanifold of $X$ such that $\pi_{i}\left(X_{t}\right)=0$ for all $i \geqq 0$. We put $X^{\prime}=X_{t_{1}}$ for a positive number $t_{1}$ which we determine in $\S 5$. The group $\Gamma$ acts isometrically, properly discontinuously, and cocompactly on $X^{\prime}$. 


\section{Horospheres.}

We take a maximal $\mathbf{Q}$-split torus $\mathbf{T}$ of $\mathbf{G}$ defined by

$$
\mathbf{T}=\rho^{-1}\left(\left\{\operatorname{diag}\left(\beta_{1}, \ldots, \beta_{n}, \ldots \ldots, \beta_{1}, \ldots, \beta_{n}\right) \mid \beta_{1} \cdots \beta_{n}=1\right\}\right) .
$$

For each $k=1, \ldots, n-1$, we define the map $\widehat{\chi}_{k}: \mathbf{T} \longrightarrow \mathbf{C}^{*}$ by

$$
\widehat{\chi}_{k}(a)=\beta_{k} \quad \text { for } a=\operatorname{diag}\left(\beta_{1}, \ldots, \beta_{n}, \ldots \ldots, \beta_{1}, \ldots, \beta_{n}\right) \in \mathbf{T},
$$

and put $\chi_{k}=\frac{\widehat{\chi}_{k}}{\widehat{\chi}_{k+1}}$. The set of $\mathbf{Q}$-roots of $\mathbf{G}$ with respect to $\mathbf{T}$ is

$$
\mathbf{Q}^{\Sigma}=\left\{\frac{\widehat{\chi}_{i}}{\widehat{\chi}_{j}} \mid i, j=1, \ldots, n ; i \neq j\right\} .
$$

We can take $\mathbf{Q}^{\Sigma^{+}}=\left\{\frac{\widehat{\chi}_{i}}{\widehat{\chi}_{j}} \mid i>j\right\}$ and $\mathbf{Q} \Delta=\left\{\chi_{1}, \ldots, \chi_{n-1}\right\}$ as a system of positive roots and simple roots respectively. Then the standard maximal parabolic Q-subgroup $\mathbf{P}_{\Theta}$ corresponding to the subset $\Theta=$ $\left\{\chi_{1}, \ldots, \chi_{n-2}\right\} \subset \mathbf{Q} \Delta$ is given by the following:

$$
\mathbf{P}_{\Theta}=\mathbf{M}_{\Theta} \mathbf{T}_{\Theta} \mathbf{U}_{\Theta}
$$

$$
\begin{aligned}
& \mathbf{T}_{\Theta}=\left(\bigcap_{\chi \in \Theta} \operatorname{ker} \chi\right)^{0} \\
& =\rho^{-1}\left(\left\{\operatorname{diag}(\underbrace{\beta, \ldots, \beta}_{n-1}, \beta^{1-n}, \ldots\right.\right. \\
& \ldots, \underbrace{\beta, \ldots, \beta}_{n-1}, \beta^{1-n}) \mid \beta \in \mathbf{C}^{*}\}) \text {, }
\end{aligned}
$$

$$
\begin{aligned}
& \mathbf{M}_{\Theta}= \\
& \rho^{-1}\left(\left\{\begin{array}{l|l}
\operatorname{diag}\left(B_{1}, b_{1}, \ldots, B_{l}, b_{l}\right) & \begin{array}{l}
B_{k} \text { is an }(n-1) \times(n-1) \text { complex } \\
\text { matrix }, b_{k} \in \mathbf{C}^{*}, \text { and }\left(\operatorname{det} B_{k}\right) b_{k}=1 \\
\text { for all } k ;\left(\operatorname{det} B_{1}\right) \cdots\left(\operatorname{det} B_{l}\right)= \pm 1
\end{array}
\end{array}\right\}\right),
\end{aligned}
$$




$$
\mathbf{U}_{\Theta}=\rho^{-1}\left(\left\{\left(\begin{array}{ccccc}
I_{n-1} & { }^{t}\left(\xi^{1}\right) & & & \\
& 1 & & & \\
& & \ddots & & \\
& & & I_{n-1} & { }^{t}\left(\boldsymbol{\xi}^{l}\right) \\
& & & & 1
\end{array}\right) \mid \begin{array}{l}
\xi^{k} \in \mathbf{C}^{n-1} \text { for } \\
k=1, \ldots, l
\end{array}\right\}\right)
$$

where $I_{n-1}$ is the $(n-1) \times(n-1)$ unit matrix. The group $\mathbf{M}_{\Theta} \mathbf{T}_{\Theta}$ is the centralizer $Z\left(\mathbf{T}_{\Theta}\right)$ of $\mathbf{T}_{\Theta}$ in $\mathbf{G}$. We put

$$
\begin{aligned}
& M_{\Theta}=\left(\left(\mathbf{M}_{\Theta}\right)_{\mathbf{R}}\right)^{0}= \\
& \rho^{-1}\left(\left\{\begin{array}{l|l}
\operatorname{diag}\left(B_{1}, b_{1}, \ldots, B_{l}, b_{l}\right) & \begin{array}{l}
B_{k} \text { is an }(n-1) \times(n-1) \text { real } \\
\text { matrix }, b_{k}>0, \text { and }\left(\operatorname{det} B_{k}\right) b_{k}=1 \\
\text { for all } k ;\left(\operatorname{det} B_{1}\right) \cdots\left(\operatorname{det} B_{l}\right)=1
\end{array}
\end{array}\right\}\right), \\
& A_{\Theta}=\left(\left(\mathbf{T}_{\Theta}\right)_{\mathbf{R}}\right)^{0} \\
& =\rho^{-1}\left(\left\{\operatorname{diag}(\underbrace{q, \ldots, q}_{n-1}, q^{1-n}, \ldots \ldots, \underbrace{q, \ldots, q}_{n-1}, q^{1-n}) \mid q>0\right\},\right) \\
& N_{\Theta}=\left(\mathbf{U}_{\Theta}\right)_{\mathbf{R}} \\
& =\rho^{-1}\left(\left\{\left(\begin{array}{ccccc}
I_{n-1} & { }^{t}\left(\xi^{1}\right) & & & \\
& 1 & & & \\
& & \ddots & & \\
& & & I_{n-1} & { }^{t}\left(\boldsymbol{\xi}^{l}\right) \\
& & & & 1
\end{array}\right) \mid \begin{array}{l}
\boldsymbol{\xi}^{k} \in \mathbf{R}^{n-1} \text { for } \\
k=1, \ldots, l
\end{array}\right\}\right) .
\end{aligned}
$$

Then $\left(\mathbf{P}_{\Theta}\right)_{\mathbf{R}}=\left(\mathbf{M}_{\Theta}\right)_{\mathbf{R}} A_{\Theta} N_{\Theta}$ is a Langlands decomposition of $\left(\mathbf{P}_{\Theta}\right)_{\mathbf{R}}$.

Since it is well known that $\mathbf{G}_{\mathbf{R}}=K\left(\mathbf{P}_{\Theta}\right)_{\mathbf{R}}$, we have $\mathbf{G}_{\mathbf{R}}=K \cdot M_{\Theta} A_{\Theta} N_{\Theta}$. Let $x_{0}$ be the coset (in $X=K \backslash \mathbf{G}_{\mathbf{R}}$ ) of the identity element. Then we have $X=x_{0} \cdot M_{\Theta} A_{\Theta} N_{\Theta}$.

We take a geodesic along the $A_{\Theta}$-orbit of $x_{0}$. Let $\mathfrak{a}_{\Theta}$ be the Lie algebra of $A_{\Theta}$. Let

$\mathrm{V}=$

$\left(d \rho_{e}\right)^{-1}\left(\frac{1}{n \sqrt{2(n-1) l}} \operatorname{diag}(-1, \ldots,-1, n-1, \ldots \ldots,-1, \ldots,-1, n-1)\right)$ $\in \mathfrak{a}_{\Theta}$, 
where $d \rho_{e}$ is the differential of $\rho$ at the identity element $e$. We define a unit speed geodesic $\gamma:[0, \infty) \longrightarrow X$ by $\gamma(t)=x_{0} \cdot(\exp t \mathrm{v})$ for $t \geqq 0$. Let $h_{\gamma}$ be the Busemann function with respect to $\gamma$ : i.e. $h_{\gamma}(x)=\lim _{t \rightarrow \infty}\left\{d_{X}(x, \gamma(t))-t\right\}$ for $x \in X$, where $d_{X}$ is the distance on $X$ ([2], [7]).

Lemma 2.1. The horosphere $h_{\gamma}^{-1}(t)$ is given by $h_{\gamma}^{-1}(t)=x_{0} \cdot a_{-t} M_{\Theta} N_{\Theta}$, where

$$
\begin{array}{r}
a_{-t}=\exp (-t \mathrm{~V})=\rho^{-1}\left(\operatorname { d i a g } \left(e^{\frac{t}{n \sqrt{2(n-1) l}}}, \ldots, e^{\frac{t}{n \sqrt{2(n-1) l}}}, e^{-\frac{t}{n} \sqrt{\frac{n-1}{2 l}}}, \ldots\right.\right. \\
\left.\left.\ldots, e^{\frac{t}{n \sqrt{2(n-1) l}}}, \ldots, e^{\frac{t}{n \sqrt{2(n-1) l}}}, e^{-\frac{t}{n} \sqrt{\frac{n-1}{2 l}}}\right)\right) .
\end{array}
$$

In particular, the horosphere through $x_{0}$ is $h_{\gamma}^{-1}(0)=x_{0} \cdot M_{\Theta} N_{\Theta}$.

Proof. Let $P(n l, \mathbf{R})$ be the set of all positive definite, symmetric matrices contained in $S L(n l, \mathbf{R})$. We identify $P(n l, \mathbf{R})$ with the symmetric space $S O(n l) \backslash S L(n l, \mathbf{R})$ in the usual way : i.e. $S L(n l, \mathbf{R})$ operates transitively on $P(n l, \mathbf{R})$ by conjugation $\left(x^{*} \cdot h^{*}={ }^{t} h^{*} x^{*} h^{*}\right.$ for $x^{*} \in P(n l, \mathbf{R}), h^{*} \in$ $S L(n l, \mathbf{R}))$, and the isotropy group of $x_{0}^{*}=I_{n l}=\operatorname{diag}(1, \ldots, 1) \in P(n l, \mathbf{R})$ is $S O(n l)$. We define a totally geodesic embedding $F: X=K \backslash \mathbf{G}_{\mathbf{R}} \longrightarrow$ $P(n l, \mathbf{R})$ by $F(K h)={ }^{t}(\rho(h))(\rho(h))$ for $h \in \mathbf{G}_{\mathbf{R}}([6],[16])$. Then the pullback of the Riemannian metric of $P(n l, \mathbf{R})$ under the map $F$ is identical with the original Riemannian metric of $X$ multiplied by $l$. Hence the speed of the geodesic $F \circ \gamma:[0, \infty) \longrightarrow P(n l, \mathbf{R})$ is $\sqrt{l}$. We define a unit speed geodesic $\gamma^{*}:[0, \infty) \longrightarrow P(n l, \mathbf{R})$ by

$$
\begin{aligned}
\gamma^{*}(t)= & F\left(\gamma\left(\frac{t}{\sqrt{l}}\right)\right)=F\left(x_{0} \cdot \exp \frac{t}{\sqrt{l}} \mathrm{~V}\right) \\
= & \operatorname{diag}\left(e^{-\frac{t}{n l} \sqrt{\frac{2}{n-1}}}, \ldots, e^{-\frac{t}{n l} \sqrt{\frac{2}{n-1}}}, e^{\frac{\sqrt{2(n-1)}}{n l} t}, \ldots\right. \\
& \left.\ldots, e^{-\frac{t}{n l} \sqrt{\frac{2}{n-1}}}, \ldots, e^{-\frac{t}{n l} \sqrt{\frac{2}{n-1}}}, e^{\frac{\sqrt{2(n-1)}}{n l} t}\right)
\end{aligned}
$$

for $t \geqq 0$. Let $h_{\gamma^{*}}^{*}$ be the Busemann function (on $P(n l, \mathbf{R})$ ) with respect to the geodesic $\gamma^{*}$. Then we have $h_{\gamma}(x)=\frac{1}{\sqrt{l}} h_{\gamma^{*}}^{*}(F(x))$ for $x \in X$. We take 
the element $\mathrm{V}^{*}$ of the tangent space $T_{x^{*}}(P(n l, \mathbf{R}))$ defined by

$$
\begin{aligned}
& \mathrm{V}^{*}=F_{*}\left(\frac{1}{\sqrt{l}} \mathrm{~V}\right)=\frac{1}{n l \sqrt{2(n-1)}} \operatorname{diag}(\underbrace{-1, \ldots,-1}_{n-1}, n-1, \ldots \\
&\ldots, \underbrace{-1, \ldots,-1}_{n-1}, n-1) .
\end{aligned}
$$

We also take the permutation $\tau$ of $(n l)$ letters defined by the following :

$$
\left\{\begin{array}{cc}
\tau(k n+b)=k(n-1)+b & \text { for } k=0,1, \ldots, l-1 \\
\tau(k n)=l(n-1)+k & \text { and } b=1, \ldots, n-1 \\
& \text { for } k=1,2, \ldots, l .
\end{array}\right.
$$

Let $A^{*}$ be the group of diagonal matrices in $S L(n l, \mathbf{R})$ with positive entries. For each $k=1, \ldots, n l-1$, we define the map $\chi_{k}^{*}: A^{*} \longrightarrow \mathbf{R}^{+}$as follows.

$$
\chi_{k}^{*}\left(a^{*}\right)=\frac{a_{k}}{a_{k+1}} \quad \text { for } a^{*}=\operatorname{diag}\left(a_{1}, \ldots, a_{n l}\right) \in A^{*} .
$$

For $x^{*}=\left(x_{i j}\right) \in P(n l, \mathbf{R})$, we denote by $\triangle_{k}\left(x^{*}\right)$, the $(k \times k)$-minor determinant in the top left corner. And for each $(n l) \times(n l)$-matrix $Z^{*}=\left(z_{i j}\right)$, we denote by $Z^{*} \cdot \tau$ the matrix $\left(z_{\tau(i) \tau(j)}\right)$.

Then, from Lemma 2.5 of [14], we obtain

$$
h_{\gamma^{*}}^{*}\left(F\left(x_{0} \cdot g\right)\right)=n l \cdot \log \prod_{k=1}^{l-1}\left\{\left(\triangle_{k}\left(F\left(x_{0} \cdot g\right) \cdot \sigma\right)\right)^{-d \chi_{k}^{*}\left(\mathrm{~V}^{*} \cdot \tau\right)}\right\},
$$

where $d \chi_{k}^{*}$ is the differential of $\chi_{k}^{*}$. We have $d \chi_{k}^{*}\left(\mathrm{~V}^{*} \cdot \tau\right) \neq 0$ if and only if $k=(n-1) l$, and then, $d \chi_{(n-1) l}^{*}\left(\mathrm{~V}^{*} \cdot \tau\right)=-\frac{1}{l \sqrt{2(n-1)}}$. Therefore,

$$
h_{\gamma}\left(x_{0} \cdot g\right)=\frac{n}{\sqrt{2(n-1) l}} \log \triangle_{(n-1) l}\left(F\left(x_{0} \cdot g\right) \cdot \tau\right) .
$$

We notice that $X=x_{0} \cdot M_{\Theta} A_{\Theta} N_{\Theta}$. Let

$$
\begin{gathered}
g_{1}=\rho^{-1}\left(\operatorname{diag}\left(B_{1}, b_{1}, \ldots B_{l}, b_{l}\right)\right) \in M_{\Theta} \\
g_{2}=\rho^{-1}\left(\operatorname{diag}\left(q, \ldots, q, q^{1-n} \ldots \ldots, q, \ldots, q, q^{1-n}\right)\right) \in A_{\Theta},
\end{gathered}
$$




$$
g_{3}=\rho^{-1}\left(\left(\begin{array}{ccccc}
I_{n-1} & \xi^{1} & & & \\
& 1 & & & \\
& & \ddots & & \\
& & & I_{n-1} & \xi^{l} \\
& & & & 1
\end{array}\right)\right) \in N_{\Theta},
$$

and $g=g_{1} g_{2} g_{3}$. Then we have

$$
F\left(x_{0} \cdot g\right) \cdot \tau=\left(\begin{array}{ccc|c}
q^{2}\left({ }^{t} B_{1} B_{1}\right) & & & \\
& \ddots & & * \\
& & q^{2}\left({ }^{t} B_{l} B_{l}\right) & \\
\hline & * * & & * * *
\end{array}\right) .
$$

So $\triangle_{(n-1) l}\left(F\left(x_{0} \cdot g\right) \cdot \tau\right)=q^{2(n-1) l}$, and $h_{\gamma}\left(x_{0} \cdot g\right)=n \sqrt{2(n-1) l} \log q$. Therefore, we obtain $h_{\gamma}^{-1}(t)=x_{0} \cdot a_{-t} M_{\Theta} N_{\Theta}$.

\section{3. $\{(n-1) l-1\}$-cycle $z_{m}$ and $(n-1) l$-chain $u_{m}$.}

The horosphere $\mathcal{L}=h_{\gamma}^{-1}(0)=x_{0} \cdot M_{\Theta} N_{\Theta}$ has a fiber bundle structure : we define the projection map $\mathcal{P}_{b}: \mathcal{L} \longrightarrow x_{0} \cdot M_{\Theta}$ by $\mathcal{P}_{b}\left(x_{0} \cdot g_{1} g_{2}\right)=$ $x_{0} \cdot g_{1}$ for $g_{1} \in M_{\Theta}$ and $g_{2} \in N_{\Theta}$. We regard $N_{\Theta}$ as $\mathbf{R}^{(n-1) l}$ by identifying $\left(y_{1}, \ldots, y_{(n-1) l}\right) \in \mathbf{R}^{(n-1) l}$ with

$$
\rho^{-1}\left(\left(\begin{array}{ccccc}
I_{n-1} & { }^{t}\left(\xi^{1}\right) & & & \\
& 1 & & & \\
& & \ddots & & \\
& & & I_{n-1} & { }^{t}\left(\xi^{l}\right) \\
& & & & 1
\end{array}\right)\right) \in N_{\Theta}
$$

where $\boldsymbol{\xi}^{k}=\left(y_{(n-1)(k-1)+1}, y_{(n-1)(k-1)+2}, \ldots, y_{(n-1) k}\right)$ for all $k$. We define a $\operatorname{map} \mathcal{P}_{f}: \mathcal{L} \longrightarrow N_{\Theta}=\mathbf{R}^{(n-1) l}$ in the direction of the fibers by $\mathcal{P}_{f}\left(x_{0} \cdot g_{1} g_{2}\right)=$ $g_{2}$ for $g_{1} \in M_{\Theta}$ and $g_{2} \in N_{\Theta}$. In this section, by using this fiber bundle structure of $\mathcal{L}$, we construct a lipschitz $\{(n-1) l-1\}$-cycle $z_{m}$ for each positive integer $m$; we also construct a $(n-1) l$-chain $u_{m}$ whose boundary is $z_{m}$.

First, we decompose the group $M_{\Theta}$. By identifying $\operatorname{diag}\left(B_{1}, \ldots, B_{l}\right)$, where $B_{1}, \ldots, B_{l} \in S L(n-1, \mathbf{C})$, with $\operatorname{diag}\left(B_{1}, 1, \ldots, B_{l}, 1\right) \in \rho(\mathbf{G})$, we can regard the direct product $(S L(n-1, \mathbf{C}))^{l}$ as a subgroup of $\rho(\mathbf{G})$. Let 
$\overline{\mathbf{G}}=\rho^{-1}\left((S L(n-1, \mathbf{C}))^{l}\right)$. Then $\overline{\mathbf{G}}$ is an algebraic Q-group. We put $\bar{K}=K \cap \overline{\mathbf{G}}$. Let $A^{\perp}$ be the center of $M_{\Theta}$ : i.e.

$$
\begin{gathered}
A^{\perp}=\rho^{-1}\left(\left\{\operatorname{diag}(\underbrace{q_{1}, \ldots, q_{1}}_{n-1},\left(q_{1}\right)^{1-n}, \ldots \ldots, \underbrace{q_{l}, \ldots, q_{l}}_{n-1},\left(q_{l}\right)^{1-n})\right.\right. \\
\left.\left.\mid q_{k}>0 \text { for all } k, q_{1} \cdots q_{l}=1\right\}\right) .
\end{gathered}
$$

Then we have $M_{\Theta}=\left(\mathbf{G}_{\mathbf{R}}\right) \cdot A^{\perp}$ and $K \cap M_{\Theta}=\bar{K}$. The space $x_{0} \cdot M_{\Theta}$ is isometric to the Riemannian direct product $(S O(n-1) \backslash S L(n-1, \mathbf{R}))^{l} \times$ $\mathbf{R}^{l-1}$, where each $S O(n-1) \backslash S L(n-1, \mathbf{R})$ (resp. $\mathbf{R}^{l-1}$ ) is equipped with the standard metric multiplied by $n /(n-1)$ (resp. the standard Euclidean metric). Let $\overline{\mathbf{P}}$ be the standard minimal parabolic $\mathbf{Q}$-subgroup of $\overline{\mathbf{G}}$ with respect to the maximal $\mathbf{Q}$-split torus $\overline{\mathbf{T}}=\mathbf{T} \cap \overline{\mathbf{G}}$ of $\overline{\mathbf{G}}$. As in the case of $\mathbf{P}_{\Theta}$ in $\S 2$, we decompose $\overline{\mathbf{P}}$ as $\overline{\mathbf{P}}=\overline{\mathbf{M}} \overline{\mathbf{T}} \overline{\mathbf{U}}$, and put

$$
\begin{aligned}
& \bar{M}=\left(\overline{\mathbf{M}}_{\mathbf{R}}\right)^{0}=\rho^{-1}\left(\left\{\operatorname{diag}\left(q_{1}^{1}, \ldots, q_{n-1}^{1}, 1, \ldots \ldots, q_{1}^{l}, \ldots, q_{n-1}^{l}, 1\right)\right.\right. \\
& \left.\left.\mid \begin{array}{l}
\text { each } q_{j}^{i} \text { is a positive real number }, \\
\prod_{j=1}^{n-1} q_{j}^{i}=1 \text { for all } i, \prod_{i=1}^{l} q_{j}^{i}=1 \text { for all } j
\end{array}\right\}\right), \\
& \bar{A}=\left(\overline{\mathbf{T}}_{\mathbf{R}}\right)^{0}=\rho^{-1}\left(\left\{\operatorname{diag}\left(q_{1}, \ldots, q_{n-1}, 1, \ldots \ldots, q_{1}, \ldots, q_{n-1}, 1\right)\right.\right. \\
& \left.\left.\mid \begin{array}{l}
\text { each } q_{j} \text { is a positive real number }, \\
q_{1} \cdots \cdots q_{n-1}=1
\end{array}\right\}\right), \\
& \bar{N}_{\Theta}=\overline{\mathbf{U}}_{\mathbf{R}}= \\
& \left.\rho^{-1}\left(\begin{array}{l|l}
\operatorname{diag}\left(\mathfrak{m}_{1}, 1, \ldots, \mathfrak{m}_{l}, 1\right) & \begin{array}{l}
\text { each } \mathfrak{m}_{k}=\left(\begin{array}{lll}
1 & & \beta_{i j}^{k} \\
& \ddots & \\
& & 1
\end{array}\right) \text { is } \\
\text { an upper triangular, real, } \\
(n-1) \times(n-1) \text { matrix }
\end{array}
\end{array}\right\}\right) .
\end{aligned}
$$

Then we have $M_{\Theta}=\left(\overline{\mathbf{G}}_{\mathbf{R}}\right) \cdot A^{\perp}=\bar{K} \bar{M} \bar{A} \bar{N} A^{\perp}$.

Let

$$
\begin{aligned}
& H_{\Theta}=\bar{M} \bar{A} A^{\perp}= \\
& =\rho^{-1}\left(\left\{\begin{array}{l|c}
\operatorname{diag}\left(q_{1}, \ldots, q_{n l}\right) & \begin{array}{c}
q_{i}>0 \text { for all } i, q_{k n+1} \cdots \cdots q_{k n+n}=1 \\
\text { for } k=0,1, \ldots, l-1, \\
q_{n} \cdot q_{2 n} \cdots \cdots q_{l n}=1
\end{array}
\end{array}\right\}\right) .
\end{aligned}
$$




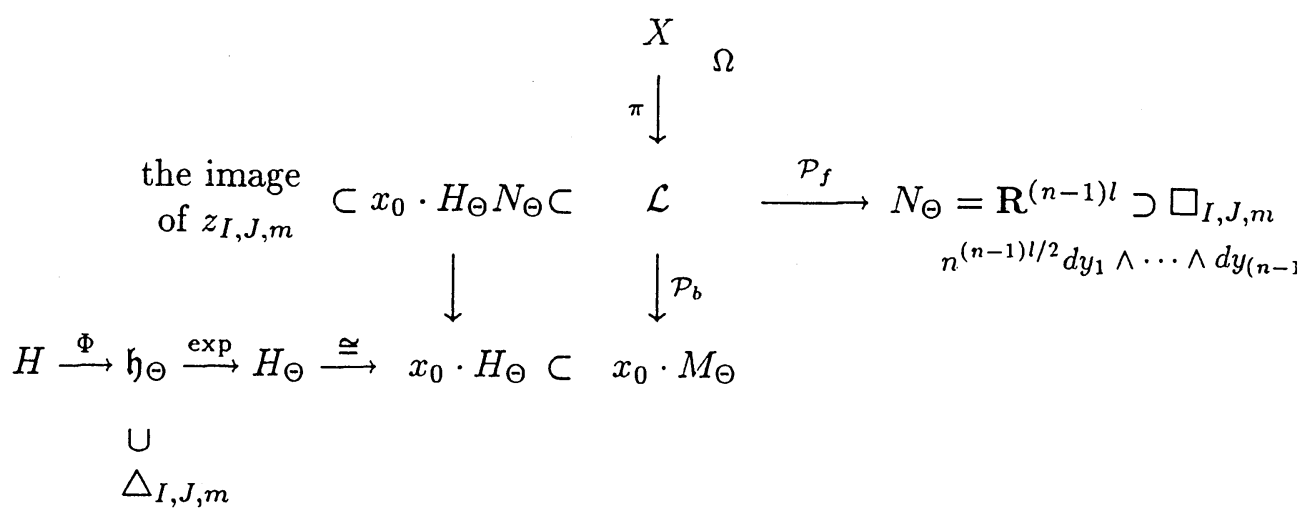

Figure 1: The construction of the chain $z_{I, J, m}$ and the form $\Omega$.

(Remark that $H_{\Theta}=M_{\Theta}$ if $n=2$.) Then the restriction of $\mathcal{P}_{b}$ to $x_{0} \cdot H_{\Theta} N_{\Theta}$ is also a fiber bundle.

From now on to Corollary 3.2 exclusive, we fix one positive integer $m$. We define $z_{m}$ as a union $\sum z_{I, J, m}$ of $\{(n-1) l-1\}$-chains, where the summation is taken over all the pair $(I, J)$ of subsets of $\{1, \ldots,(n-1) l\}$ such that $I \cap J$ is the empty set $\phi$ and that $I \cup J \neq \phi$. We fix one such pair $(I, J)$ and define the $\{(n-1) l-1\}$-chain $z_{I, J, m}$ as follows (see Figure 1$)$.

Let $\mathfrak{h}_{\Theta}$ be the Lie algebra of $H_{\Theta}$ and

$$
H=\left\{\left(h_{1}, \ldots, h_{(n-1) l}\right) \in \mathbf{R}^{(n-1) l} \mid h_{1}+\cdots+h_{(n-1) l}=0\right\}
$$

be a hyperplane in $\mathbf{R}^{(n-1) l}$. We define a map $\Phi: H \longrightarrow \mathfrak{h}_{\Theta}$ by

$$
\begin{aligned}
& \Phi\left(\left(h_{1}, \ldots, h_{(n-1) l}\right)\right)= \\
& \rho^{-1}\left(\operatorname { d i a g } \left(h_{1}, \ldots, h_{n-1},-\left(h_{1}+\cdots+h_{n-1}\right), \ldots\right.\right. \\
& \quad \ldots, h_{(n-1)(k-1)+1}, \ldots, h_{(n-1) k},-\left(h_{(n-1)(k-1)+1}+\cdots+h_{(n-1) k}\right), \ldots \\
& \left.\left.\quad \ldots, h_{(n-1)(l-1)+1}, \ldots, h_{(n-1) l},-\left(h_{(n-1)(l-1)+1}+\cdots+h_{(n-1) l}\right)\right)\right) .
\end{aligned}
$$

This map $\Phi$ is a lipschitz diffeomorphism when we give $\mathfrak{h}_{\Theta}$ the inner product induced from the Killing form of the Lie algebra of $\mathbf{G}_{\mathbf{R}}$. We define $(n-1) l$ points $w_{1, m}, \ldots, w_{(n-1) l, m}$ of $H$ as follows : for each $k=0, \ldots, l-1$ and 


$$
\begin{aligned}
& j=1, \ldots, n-1, \text { let } \\
& \begin{aligned}
& w_{(n-1) k+j, m} \\
&=(-m, \ldots \ldots .,-m,-\{(n-1) l+1\} m, \ldots,-\{(n-1) l+1\} m \\
&\left\{(n-1)^{2} l-1\right\} m,-\{(n-1) l+1\} m, \ldots,-\{(n-1) l+1\} m, \\
&-m, \ldots \ldots . .-m),
\end{aligned}
\end{aligned}
$$

where the $i$-th entry for $(n-1) k+1 \leqq i \leqq(n-1)(k+1)$ with $i \neq(n-1) k+j$ is $-\{(n-1) l+1\} m$, the $\{(n-1) k+j\}$-th entry is $\left\{(n-1)^{2} l-1\right\} m$, and the rests are all equal to $-m$. We put $v_{i, m}=\Phi\left(w_{i, m}\right)$ for $i=1, \ldots,(n-1) l$.

Let $I \cup J=\left\{i_{1}, \ldots, i_{s}\right\}: i_{1}<\ldots<i_{s}$. We denote by $\triangle_{I, J, m}$ the simplex whose vertices are $v_{i_{1}, m}, \ldots, v_{i_{s}, m}$ : i.e. $\triangle_{I, J, m}=\left|v_{i_{1}, m} \cdots \cdots v_{i_{s}, m}\right|$. Let $\square_{I, J, m}$ be the $\{(n-1) l-s\}$-dimensional cube in $N_{\Theta}=\mathbf{R}^{(n-1) l}$ defined by

$$
\begin{aligned}
& \square_{I, J, m}= \\
& \left\{\begin{array}{l|l}
\left(y_{1}, \ldots, y_{(n-1) l}\right) \in \mathbf{R}^{(n-1) l}=N_{\Theta} & \begin{array}{l}
y_{i}=e^{n m} \text { if } i \in I \\
y_{j}=-e^{n m} \text { if } j \in J \\
-e^{n m} \leqq y_{k} \leqq e^{n m} \text { if } k \notin I \cup J
\end{array}
\end{array}\right\} .
\end{aligned}
$$

We define the map $z_{I, J, m}: \triangle_{I, J, m} \times \square_{I, J, m} \longrightarrow x_{0} \cdot H_{\Theta} N_{\Theta}$ by $z_{I, J, m}(v, y)=$ $x_{0} \cdot(\exp v) y$ for $v \in \triangle_{I, J, m}, y \in \square_{I, J, m}$. By considering the standard simplicial decomposition of $\triangle_{I, J, m} \times \square_{I, J, m}$, we regard the map $z_{I, J, m}$ as an $\{(n-1) l-1\}$-chain (see Figure 2).

Lemma 3.1. The image of $z_{I, J, m}$ (equipped with the induced metric from $X)$ is isometric to the direct product of the simplex $\left|v_{i_{1}, m} \cdots v_{i_{s}, m}\right|$ and $[-\sqrt{n}, \sqrt{n}]^{(n-1) l-s}$.

Proof. Any $C^{\infty}$-curve $c:(-\delta, \delta) \longrightarrow x_{0} \cdot H_{\Theta} N_{\Theta}(\delta>0)$ in the image of $z_{I, J, m}$ can be written as $c(t)=x_{0} \cdot \widehat{c}(t)$ by a curve $\widehat{c}:(-\delta, \delta) \longrightarrow H_{\Theta} N_{\Theta}$ in $H_{\Theta} N_{\Theta}$ such that

$$
\begin{aligned}
& \rho(\widehat{c}(t))=\operatorname{diag}\left(e^{b_{1}^{1}(t)}, \ldots, e^{b_{n}^{1}(t)}, \ldots \ldots, e^{b_{1}^{l}(t)}, \ldots, e^{b_{n}^{l}(t)}\right) \\
& \times\left(\begin{array}{ccccc}
I_{n-1} & { }^{t}\left(\xi^{1}(t)\right) & & & \\
& 1 & & & \\
& & \ddots & & \\
& & & I_{n-1} & \\
& & & \\
& & \left.\xi^{l}(t)\right) \\
& & & & 1
\end{array}\right)
\end{aligned}
$$



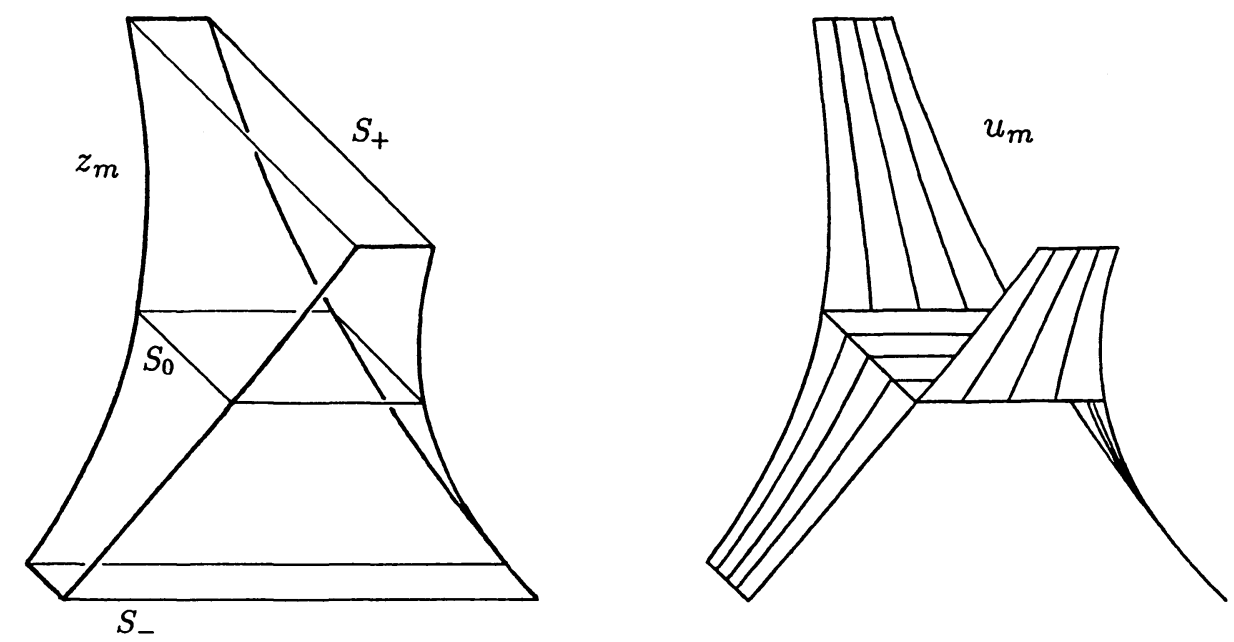

Figure 2: $(n=l=2)$

for $t \in(-\delta, \delta)$, where all $\xi^{k}(t)=\left(\xi_{1}^{k}(t), \ldots, \xi_{n-1}^{k}(t)\right)\left(\right.$ resp. $\left.b_{j}^{k}(t)\right)$ are $C^{\infty}$ maps $(-\delta, \delta) \longrightarrow \mathbf{R}^{n-1}$ (resp. $C^{\infty}$-functions on $(-\delta, \delta)$ ), and $\sum_{i=1}^{n} b_{i}^{k} \equiv 0$ for all $k$. Since the right translations are isometries, we take the following element $g$ of $H_{\Theta} N_{\Theta}$ and put $\widetilde{c}(t)=c(t) \cdot g=x_{0} \cdot \widehat{c}(t) g$.

$$
\begin{aligned}
& \rho(g)=\operatorname{diag}\left(e^{-b_{1}^{1}(0)}, \ldots, e^{-b_{n}^{1}(0)}, \ldots \ldots, e^{-b_{1}^{l}(0)}, \ldots, e^{-b_{n}^{l}(0)}\right) \\
& \times\left(\begin{array}{ccccc}
I_{n-1} & { }^{t} \zeta^{1} & & & \\
& 1 & & & \\
& & \ddots & & \\
& & & I_{n-1} & { }^{t} \zeta^{l} \\
& & & & 1
\end{array}\right), \\
& \zeta^{k}=\left(-e^{b_{1}^{k}(0)-b_{n}^{k}(0)} \xi_{1}^{k}(0), \ldots-e^{b_{n-1}^{k}(0)-b_{n}^{k}(0)} \xi_{n-1}^{k}(0)\right) \text { for } k=1, \ldots, l .
\end{aligned}
$$

We remark that $\widetilde{c}:(-\delta, \delta) \longrightarrow x_{0} \cdot H_{\Theta} N_{\Theta}$ is a curve such that $\widetilde{c}(0)=x_{0}$. Since

$$
\widehat{c}(t) g=\rho^{-1}\left(\left(\begin{array}{ccccc}
B^{1}(t) & t\left(\eta^{1}(t)\right) & & & \\
& e^{b_{n}^{1}(t)-b_{n}^{1}(0)} & & & \\
& & \ddots & & \\
& & & B^{l}(t) & c^{t}\left(\eta^{l}(t)\right) \\
& & & & e^{b_{n}^{l}(t)-b_{n}^{l}(0)}
\end{array}\right)\right)
$$


where,

$$
B^{k}(t)=\operatorname{diag}\left(e^{b_{1}^{k}(t)-b_{1}^{k}(0)}, \ldots, e^{b_{n-1}^{k}(t)-b_{n-1}^{k}(0)}\right)
$$

and

$$
\eta^{k}(t)=\left(e^{b_{1}^{k}(t)-b_{n}^{k}(0)}\left(\xi_{1}^{k}(t)-\xi_{1}^{k}(0)\right), \ldots, e^{b_{n-1}^{k}(t)-b_{n}^{k}(0)}\left(\xi_{n-1}^{k}(t)-\xi_{n-1}^{k}(0)\right)\right)
$$

for $k=1, \ldots, l$, we have

$$
\begin{aligned}
& \left.\frac{d}{d t}(\widehat{c}(t) g)\right|_{t=0}= \\
& \left(d \rho_{e}\right)^{-1}\left(\left(\begin{array}{ccccc}
\left(B^{1}\right)^{\prime}(0) & t\left\{\left(\eta^{1}\right)^{\prime}(0)\right\} & & & \\
& \left(b_{n}^{1}\right)^{\prime}(0) & & & \\
& & \ddots & & \\
& & & \left(B_{l}\right)^{\prime}(0) & t\left\{\left(\boldsymbol{\eta}^{l}\right)^{\prime}(0)\right\} \\
& & & & \left(b_{n}^{l}\right)^{\prime}(0)
\end{array}\right)\right)
\end{aligned}
$$

and

$$
\left(\boldsymbol{\eta}^{k}\right)^{\prime}(0)=\left(e^{b_{1}^{k}(0)-b_{n}^{k}(0)}\left(\xi_{1}^{k}\right)^{\prime}(0), \ldots, e^{b_{n-1}^{k}(0)-b_{n}^{k}(0)}\left(\xi_{n-1}^{k}\right)^{\prime}(0)\right)
$$

for $k=1, \ldots, l$. Let $\mathfrak{g}$ (resp. $\mathfrak{k}$ ) be the Lie algebra of $\mathbf{G}_{\mathbf{R}}$ (resp. $K$ ). We identify the tangent space $T_{x_{0}}(X)$ with the orthogonal complement $\mathfrak{p}$ of $\mathfrak{k}$ in $\mathfrak{g}$ with respect to the Killing form of $\mathfrak{g}$. Under this identification, we can express the tangent vector $\dot{\tilde{c}}(0)=\mathrm{v}$ as follows:

$$
\begin{gathered}
\mathrm{v}=\mathrm{v}_{1}+\mathrm{v}_{2} ; \mathrm{v}_{1} \in T_{x_{0}}\left(x_{0} \cdot M_{\Theta}\right), \mathrm{v}_{2} \in T_{x_{0}}\left(x_{0} \cdot N_{\Theta}\right), \\
\mathrm{v}_{1}=\left(d \rho_{e}\right)^{-1}\left(\operatorname{diag}\left(\left(b_{1}^{1}\right)^{\prime}(0), \ldots,\left(b_{n}^{1}\right)^{\prime}(0), \ldots \ldots\left(b_{1}^{l}\right)^{\prime}(0), \ldots,\left(b_{n}^{l}\right)^{\prime}(0)\right)\right), \\
\mathbf{v}_{2}=\left(d \rho_{e}\right)^{-1}\left(\left(\begin{array}{cccc}
0 & \frac{t\left(\left(\eta^{1}\right)^{\prime}(0)\right)}{2} & \\
\frac{\left(\eta^{1}\right)^{\prime}(0)}{2} & 0 & & \\
& & \ddots & \\
& & 0 & \frac{{ }^{t}\left(\left(\eta^{l}\right)^{\prime}(0)\right)}{2} \\
& & \frac{\left(\eta^{l}\right)^{\prime}(0)}{2} & 0
\end{array}\right)\right) .
\end{gathered}
$$

We remark that two subspaces $T_{x_{0}}\left(x_{0} \cdot M_{\Theta}\right)$ and $T_{x_{0}}\left(x_{0} \cdot N_{\Theta}\right)$ are orthogonal to each other in $T_{x_{0}}(X)$. Let $\varphi:(-\delta, \delta) \longrightarrow x_{0} \cdot N_{\Theta}$ be the curve given by

$$
\varphi(t)=x_{0} \cdot\left(\rho^{-1}\left(\left(\begin{array}{ccccc}
I_{n-1} & { }^{t}\left(\xi^{1}(t)\right) & & & \\
& 1 & & & \\
& & \ddots & & \\
& & & I_{n-1} & { }^{t}\left(\xi^{1}(t)\right) \\
& & & & 1
\end{array}\right)\right)\right.
$$


for $t \in(-\delta, \delta)$.

Assertion. Let $i=(n-1) k+j: 0 \leqq k \leqq l-1,1 \leqq j \leqq n-1$.

(1) If $i \notin I \cup J$, then we have $b_{j}^{k}(t)-b_{n}^{k}(t)=-n m$ for all $t \in(-\delta, \delta)$.

(2) If $i \in I \cup J$, then $\left(\xi_{j}^{k}\right)^{\prime}(0)=0$.

Proof of Assertion. Since (2) is an immediate consequence of the definition of $\xi_{j}^{k}$, we prove (1).

(case 1) $(n-1) k+\nu \notin I \cup J$ for all $\nu \in\{1, \ldots, n-1\}-\{j\}$

We have $b_{\mu}^{k}(t)=-m$ for all $\mu \in\{1, \ldots, n-1\}$ and all $t$. So $b_{n}^{k} \equiv$ $(n-1) m$, and $b_{j}^{k}-b_{n}^{k} \equiv-n m$.

(case 2 ) $(n-1) k+\nu \in I \cup J$ for some $\nu \in\{1, \ldots, n-1\}-\{j\}$

We can take the smooth function $\lambda:(-\delta, \delta) \longrightarrow[0,1]$ such that

$b_{j}^{k}(t)=\lambda(t)[-\{(n-1) l+1\} m]+(1-\lambda(t))(-m)=-\{\lambda(t)(n-1) l+1\} m$.

For each $\mu \in\{1, \ldots, n-1\}-\{j\}$ with $(n-1) k+\mu \in I \cup J$, the $(n k)-(n k)$ entry of $v_{(n-1) k+\mu, m}=\Phi\left(w_{(n-1) k+\mu, m}\right)$ is equal to

$$
-(n-2)[-\{(n-1) l+1\} m]-\left\{(n-1)^{2} l-1\right\} m=-(n l-l-n+1) m .
$$

And for each $k^{\prime} \neq k$ and $\mu^{\prime} \in\{1, \ldots, n-1\}$, the $(n k)-(n k)$ entry of $v_{(n-1) k^{\prime}+\mu^{\prime}, m}$ is equal to $(n-1) m$. So we have

$$
\begin{aligned}
b_{n}^{k}(t)=\lambda(t)\{-(n l-l-n+1) m\}+ \\
+(1-\lambda(t))(n-1) m=-\{\lambda(t)(n-1) l+1-n\} m .
\end{aligned}
$$

Hence, $b_{j}^{k}(t)-b_{n}^{k}(t)=-n m$ for all $t$.

By this assertion, $\left\|\mathrm{v}_{2}\right\|=e^{-n m}\|\dot{\varphi}(0)\|$. When we identify $N_{\Theta}=\mathbf{R}^{(n-1) l}$ with $x_{0} \cdot N_{\Theta}$ in the natural way, the metric of $\mathbf{R}^{(n-1) l}$ induced from $X$ is given by $n\left(d y_{1}^{2}+\cdots+d y_{(n-1) l}^{2}\right)$. Therefore, the image of $z_{I, J, m}$ is isometric to $\left|v_{i_{1}, m} \cdots v_{i_{s}, m}\right| \times[-\sqrt{n}, \sqrt{n}]^{(n-1) l-s}$.

From the above lemma, the $\{(n-1) l-1\}$-chain $z_{I, J, m}$ is lipschitz.

Let $z_{m}=\sum z_{I, J, m}$, where the summation is taken over all the pair $(I, J)$ of subsets of $\{1, \ldots,(n-1) l\}$ such that $I \cup J \neq \phi$ and that $I \cap J=$ $\phi$. As is shown in [5, pp. 239-240], $\sum\left(\triangle_{I, J, m} \times \square_{I, J, m}\right)$ is topologically 
the $\{(n-1) l-1\}$-dimensional sphere $S^{(n-1) l-1}$. Hence, $z_{m}$ is a lipschitz $\{(n-1) l-1\}$-cycle. The following is an immediate consequence of Lemma 3.1.

Corollary 3.2. The $\{(n-1) l-1\}$-dimensional volume $\operatorname{mass}_{(n-1) l-1}\left(z_{m}\right)$ and the diameter $\operatorname{diam}\left(z_{m}\right)$ of $z_{m}$ are bounded above by some polynomials in $m$.

We again fix one positive integer $m$, and define an $(n-1) l$-chain $u_{m}$ which bounds $z_{m}$ as follows. For each pair $(I, J)$ of subsets of $\{1, \ldots,(n-1) l\}$ such that $I \cup J \neq \phi, I \cap J=\phi$, we define a map $u_{I, J, m}^{\prime}: \triangle_{I, J, m} \times \square_{I, J, m} \times[0,1] \longrightarrow$ $x_{0} \cdot H_{\Theta} N_{\Theta}$ by $u_{I, J, m}^{\prime}(v, y, t)=x_{0} \cdot(\exp t v) y$ for $v \in \triangle_{I, J, m}, y \in \square_{I, J, m}$, and $t \in[0,1]$. As before, we regard the map $u_{I, J, m}^{\prime}$ as a lipschitz $(n-1) l$-chain, and put $u_{m}^{\prime}=\sum_{I, J} u_{I, J, m}^{\prime}$. We put

$$
\square_{m}=\left\{\begin{array}{l|l}
\left(y_{1}, \ldots, y_{(n-1) l}\right) \in N_{\Theta}=\mathbf{R}^{(n-1) l} & \begin{array}{c}
-e^{n m} \leqq y_{k} \leqq e^{n m} \\
\text { for all } k
\end{array}
\end{array}\right\},
$$

and define a map $u_{m}^{\prime \prime}: \square_{m} \longrightarrow x_{0} \cdot H_{\Theta} N_{\Theta}$ by $u_{m}^{\prime \prime}(y)=x_{0} \cdot y$ for $y \in \square_{m}$. Let $u_{m}=u_{m}^{\prime}+u_{m}^{\prime \prime}$. Then $u_{m}$ is a lipschitz $(n-1) l$-chain which bounds $z_{m}$ (see Figure 2).

\section{Closed $(n-1) l$-form $\Omega$ and its norm.}

We identify $\mathbf{R}^{(n-1) l}$ with $N_{\Theta}$ as in $\S 3$. We also identify $N_{\Theta}$ with $x_{0} \cdot N_{\Theta}$ in the natural way. Then the metric of $\mathbf{R}^{(n-1) l}$ induced from $X$ is $n\left(d y_{1}^{2}+\right.$ $\left.\cdots+d y_{(n-1) l}^{2}\right)$, and the volume form of $\mathbf{R}^{(n-1) l}$ is $n^{(n-1) l / 2} d y_{1} \wedge \cdots \wedge d y_{(n-1) l}$. Let $\pi: X \longrightarrow \mathcal{L}=x_{0} \cdot M_{\Theta} N_{\Theta}$ be the map given by $\pi\left(\gamma(t) \cdot g_{1} g_{2}\right)=x_{0} \cdot g_{1} g_{2}$ for $t \in \mathbf{R}, g_{1} \in M_{\Theta}$, and $g_{2} \in N_{\Theta}$. Let $\Omega$ be the pullback of the $(n-1) l$-form $n^{(n-1) l / 2} d y_{1} \wedge \cdots \wedge d y_{(n-1) l}$ on $\mathbf{R}^{(n-1) l}$ under the map $\mathcal{P}_{f} \circ \pi: X \longrightarrow N_{\Theta}=$ $\mathbf{R}^{(n-1) l}$ (see Figure 1). (Notice that $\left(\mathcal{P}_{f} \circ \pi\right)\left(\gamma(t) \cdot g_{1} g_{2}\right)=g_{2}$ for $t \in \mathbf{R}$, $g_{1} \in M_{\Theta}$, and $g_{2} \in N_{\Theta}$.)

For $x \in X$ and $\mathrm{v}_{1}, \ldots, \mathrm{v}_{(n-1) l} \in T_{x}(X)$, we denote by $\mathcal{V}\left(\mathrm{v}_{1}, \ldots, \mathrm{v}_{(n-1) l}\right)$ the $(n-1) l$-dimensional volume of parallelotope spanned by $\mathrm{v}_{1}, \ldots, \mathrm{v}_{(n-1) l}$.

Definition 4.1. We define the norm $\|\Omega\|_{x}$ of $\Omega$ at $x \in X$ to be

$$
\|\Omega\|_{x}=\sup \left\{\frac{\left|\Omega\left(\mathrm{v}_{1}, \ldots, \mathrm{v}_{(n-1) l}\right)\right|}{\mathcal{V}\left(\mathrm{v}_{1}, \ldots, \mathrm{v}_{(n-1) l}\right)} \mid \begin{array}{l}
\mathrm{v}_{1}, \ldots, \mathrm{v}_{(n-1) l} \in T_{x}(X) \\
\mathcal{V}\left(\mathrm{v}_{1}, \ldots, \mathrm{v}_{(n-1) l} \neq \neq 0\right.
\end{array}\right\} .
$$


Lemma 4.2. $\|\Omega\|_{x}=\exp \left(\sqrt{\frac{(n-1) l}{2}} t\right) \quad$ for all $x \in h_{\gamma}^{-1}(-t)$.

Proof. Let $M_{\Theta}=\bar{K} \bar{M} \bar{A} \bar{N} A^{\perp}$ as in $\S 3$. As in Lemma 2.1, we put

$$
\begin{array}{r}
a_{t}=\exp (t \mathrm{~V})=\rho^{-1}\left(\operatorname { d i a g } \left(e^{-\frac{t}{n \sqrt{2(n-1) l}}}, \ldots, e^{-\frac{t}{n \sqrt{2(n-1) l}}}, e^{\frac{t}{n} \sqrt{\frac{n-1}{2 l}}}, \ldots\right.\right. \\
\left.\left.\ldots, e^{-\frac{t}{n \sqrt{2(n-1) l}}}, \ldots, e^{-\frac{t}{n \sqrt{2(n-1) l}}}, e^{\frac{t}{n} \sqrt{\frac{n-1}{2 l}}}\right)\right)
\end{array}
$$

for $t \in \mathbf{R}$. For $\mathbf{y}=\left(y_{1}, \ldots, y_{(n-1) l}\right) \in \mathbf{R}^{(n-1) l}$, we put

$$
n_{\mathbf{y}}=\rho^{-1}\left(\left(\begin{array}{ccccc}
I_{n-1} & { }^{t}\left(\xi^{1}\right) & & & \\
& 1 & & & \\
& & \ddots & & \\
& & & I_{n-1} & { }^{t}\left(\xi^{l}\right) \\
& & & & 1
\end{array}\right)\right) \in N_{\Theta}
$$

where $\xi^{k}=\left(y_{(n-1)(k-1)+1}, y_{(n-1)(k-1)+2}, \ldots, y_{(n-1) k}\right)$ for each $k$. Notice that $x_{0} \cdot M_{\Theta}$ is diffeomorphic to $\bar{M} \bar{A} \bar{N} A^{\perp}$. So we can take a diffeomorphism $\Psi: \mathbf{R}^{\left(n^{2}-n\right) l / 2-1} \times \mathbf{R} \times \mathbf{R}^{(n-1) l} \longrightarrow X$ such that $\Psi(\boldsymbol{r}, t, \mathbf{y})=x_{0} \cdot \psi(\boldsymbol{r}) a_{t} n_{\mathbf{y}}$ for $\boldsymbol{r}=\left(r_{1}, \ldots, r_{\left(n^{2}-n\right) l / 2-1}\right) \in \mathbf{R}^{\left(n^{2}-n\right) l / 2-1}, t \in \mathbf{R}$, and $\mathbf{y}=\left(y_{1}, \ldots, y_{(n-1) l}\right) \in$ $\mathbf{R}^{(n-1) l}$, where $\psi: \mathbf{R}^{\left(n^{2}-n\right) l / 2-1} \longrightarrow \bar{M} \bar{A} \bar{N} A^{\perp}$ is a diffeomorphism with $\psi(0)=I_{n l}$. Suppose that $x=x_{0} \cdot \psi(\boldsymbol{r}) a_{t} n_{\mathbf{y}}=x_{0} \cdot n_{\mathbf{y}^{\prime}} \psi(\boldsymbol{r}) a_{t}, \mathbf{y}^{\prime}=$ $\left(y_{1}^{\prime}, \cdots, y_{(n-1) l}^{\prime}\right)$, and

$$
\psi(\boldsymbol{r})=\operatorname{diag}\left(B_{1}, b_{1}, \ldots, B_{l}, b_{l}\right) \in \bar{M} \bar{A} \bar{N} A^{\perp},
$$

where each $B_{k}$ is an $(n-1) \times(n-1)$ matrices. Then the relation between $\mathbf{y}$ and $\mathbf{y}^{\prime}$ is given by the following :

$$
{ }^{t} \mathbf{y}=e^{\frac{t}{\sqrt{2(n-1) l}}}\left(\begin{array}{lll}
b_{1} B_{1}^{-1} & & \\
& \ddots & \\
& & b_{l} B_{l}^{-1}
\end{array}\right){ }^{t}\left(\mathbf{y}^{\prime}\right)
$$

Let

$$
\widetilde{e}_{i}=\left.\frac{d}{d \kappa}\left\{x_{0} \cdot n_{\left(y_{1}^{\prime}, \ldots, y_{i-1}^{\prime}, y_{i}^{\prime}+\kappa, y_{i+1}^{\prime}, \ldots, y_{(n-1) l}^{\prime}\right)} \psi(\boldsymbol{r}) a_{t}\right\}\right|_{\kappa=0}
$$

and $e_{i}=\widetilde{e}_{i} /\left\|\tilde{e}_{i}\right\|$ for $i=1, \ldots,(n-1) l$. Then $\left\{e_{1}, \ldots, e_{(n-1) l}\right\}$ is an orthonormal basis of the tangent space $T_{x}\left(x_{0} \cdot \psi(r) a_{t} N_{\Theta}\right)$. We put

$$
\widehat{e}_{i}=\left(\frac{\partial}{\partial y_{i}}\right)_{\mathrm{y}} /\left\|\left(\frac{\partial}{\partial y_{i}}\right)_{\mathrm{y}}\right\| \quad \text { for } \quad i=1, \ldots,(n-1) l \text {. }
$$


Under the identification $\mathbf{R}^{(n-1) l}=x_{0} \cdot N_{\Theta},\left\{\widehat{e}_{1}, \ldots, \widehat{e}_{(n-1) l}\right\}$ is an orthonormal basis of the tangent space $T_{x_{0} \cdot n_{y}}\left(x_{0} \cdot N_{\Theta}\right)$. The restriction of the differential $\left(\mathcal{P}_{f} \circ \pi\right)_{* x}$ of the map $\mathcal{P}_{f} \circ \pi: X \longrightarrow N_{\Theta}=\mathbf{R}^{(n-1) l}$ to the tangent space $T_{x}\left(x_{0} \cdot M_{\Theta} A_{\Theta} n_{\mathrm{y}}\right)$ is the zero map. And the matrix of the restriction $\left.\left(\mathcal{P}_{f} \circ \pi\right)_{*}\right|_{T_{x}\left(x_{0} \cdot \psi(\boldsymbol{r}) a_{t} N_{\Theta}\right)}: T_{x}\left(x_{0} \cdot \psi(\boldsymbol{r}) a_{t} N_{\Theta}\right) \longrightarrow T_{x_{0} \cdot n_{\mathbf{y}}}\left(x_{0} \cdot N_{\Theta}\right)$ of the linear $\operatorname{map}\left(\mathcal{P}_{f} \circ \pi\right)_{*}$ to $T_{x}\left(x_{0} \cdot \psi(r) a_{t} N_{\Theta}\right)$ with respect to the basis $\left\{e_{1}, \ldots, e_{(n-1) l}\right\}$ and $\left\{\widehat{e}_{1}, \ldots, \widehat{e}_{(n-1) l}\right\}$ is

$$
Q=\left(q_{i j}\right)=e^{\frac{t}{\sqrt{2(n-1) l}}}\left(\begin{array}{lll}
b_{1} B_{1}^{-1} & & \\
& \ddots & \\
& & b_{l} B_{l}^{-1}
\end{array}\right) .
$$

We suppose that $\mathcal{V}\left(\mathrm{v}_{1}, \ldots, \mathrm{v}_{(n-1) l}\right) \neq 0$. Let $\left\{\mathrm{w}_{1}, \ldots, \mathrm{w}_{(n-1) l}\right\}$ be an orthonormal basis of the subspace $\mathbf{R v}_{1}+\cdots+\mathbf{R}_{(n-1) l}$ of $T(X)_{x}$. Each $\mathrm{v}_{k}$ is expressed as $\mathrm{v}_{k}=\sum_{j=1}^{(n-1) l} \xi_{k j} \mathrm{w}_{j}$, where every $\xi_{k j}$ is a real number, and then, $\mathcal{V}\left(\mathrm{v}_{1}, \ldots, \mathrm{v}_{(n-1) l}\right)=\left|\operatorname{det}\left(\xi_{k j}\right)\right|$. For each $k$, we can write $\mathrm{w}_{k}=\mathrm{w}^{\prime}{ }_{k}+\mathrm{w}^{\prime \prime}{ }_{k}, \mathrm{w}^{\prime \prime}{ }_{k}=\sum_{i=1}^{(n-1) l} \eta_{k i} e_{i}$, where every $\eta_{k i}$ is a real number and $\mathrm{w}^{\prime}{ }_{k} \perp e_{i}$ for all $i$. (Remark that $\left\|\mathrm{w}^{\prime \prime}{ }_{k}\right\| \leqq\left\|\mathrm{w}_{k}\right\|=1$.) Noticing that $T_{x}\left(x_{0} \cdot M_{\Theta} A_{\Theta} n_{\mathrm{y}}\right) \perp T_{x}\left(x_{0} \cdot \psi(\boldsymbol{r}) a_{t} N_{\Theta}\right)$, we put $\mathrm{v}_{k}=\mathrm{v}^{\prime}{ }_{k}+\mathrm{v}^{\prime \prime}{ }_{k}$ : $\mathrm{v}^{\prime}{ }_{k} \in T_{x}\left(x_{0} \cdot M_{\Theta} A_{\Theta} n_{\mathbf{y}}\right), \mathrm{v}^{\prime \prime}{ }_{k} \in T_{x}\left(x_{0} \cdot \psi(r) a_{t} N_{\Theta}\right)$. Then

$$
\mathrm{v}^{\prime \prime}{ }_{k}=\sum_{j=1}^{(n-1) l} \xi_{k j \mathrm{w}}{ }_{j}{ }_{j}=\sum_{j=1}^{(n-1) l} \xi_{k j}\left(\sum_{\nu=1}^{(n-1) l} \eta_{j \nu} e_{\nu}\right) .
$$

Let $\left(\zeta_{i j}\right)=\left(\xi_{i j}\right)\left(\eta_{i j}\right)$. Then $\mathrm{v}^{\prime \prime}{ }_{k}=\sum_{\nu=1}^{(n-1) l} \zeta_{k \nu} e_{\nu}$. Hence

$$
\begin{aligned}
\left(\mathcal{P}_{f} \circ \pi\right)_{*}\left(\mathrm{v}_{k}\right) & =\left(\mathcal{P}_{f} \circ \pi\right)_{*}\left(\mathrm{v}^{\prime \prime}{ }_{k}\right)=\sum_{i=1}^{(n-1) l} \zeta_{k i}\left(\mathcal{P}_{f} \circ \pi\right)_{*}\left(e_{i}\right) \\
& =\sum_{i=1}^{(n-1) l} \zeta_{k i}\left(\sum_{\nu=1}^{(n-1) l} q_{\nu i} \widehat{e}_{\nu}\right)=\sum_{\nu=1}^{(n-1) l}\left(\sum_{i=1}^{(n-1) l} \zeta_{k i} q_{\nu i}\right) \widehat{e}_{\nu}
\end{aligned}
$$

and

$$
\Omega\left(\mathrm{v}_{1}, \ldots, \mathrm{v}_{(n-1) l}\right)=\operatorname{det}\left(\left(\zeta_{i j}\right)^{t} Q\right)=e^{\sqrt{(n-1) l / 2} t} \cdot \operatorname{det}\left(\xi_{i j}\right) \cdot \operatorname{det}\left(\eta_{i j}\right) .
$$

We have

$$
\frac{\left|\Omega\left(\mathrm{v}_{1}, \ldots, \mathrm{v}_{(n-1) l}\right)\right|}{\mathcal{V}\left(\mathrm{v}_{1}, \ldots, \mathrm{v}_{(n-1) l}\right)}=e^{\sqrt{(n-1) l / 2} t} \cdot\left|\operatorname{det}\left(\eta_{i j}\right)\right|
$$




$$
\leqq e^{\sqrt{(n-1) l / 2} t} \cdot\left\|\mathrm{w}^{\prime \prime}{ }_{1}\right\| \cdots \cdot\left\|^{\prime \prime}{ }_{(n-1) l}\right\| \leqq e^{\sqrt{(n-1) l / 2} t}
$$

Since the equality holds when $\mathrm{v}^{\prime}{ }_{k}=0$ for all $k$, we obtain $\|\Omega\|_{x}=$ $e^{\sqrt{(n-1) l / 2} t}$.

\section{Isoperimetric inequality on the complement of a horoball.}

Let $\Pi: X \longrightarrow X / \Gamma^{\prime}$ be the projection. If $n=2, H_{\Theta}=M_{\Theta}$. And in this case, it is known that $\Pi\left(x_{0} \cdot H_{\Theta} N_{\Theta}\right)$ is compact (see $\left.[15, \S 1]\right)$. In the case $n \geqq 3, \Pi\left(x_{0} \cdot H_{\Theta} N_{\Theta}\right)$ is not compact. In fact, we can show that the geodesic $\Pi \circ \gamma^{\prime}:[0, \infty) \longrightarrow X / \Gamma^{\prime}$ is a ray (i.e. $d_{X / \Gamma^{\prime}}\left(\Pi \circ \gamma^{\prime}(t), \Pi \circ \gamma^{\prime}\left(t^{\prime}\right)\right)=\left|t-t^{\prime}\right|$ for all $\left.t, t^{\prime} \geqq 0\right)$ for the following geodesic $\gamma^{\prime}:[0, \infty) \longrightarrow X$ by the same argument in $\S 2$ of [14].

$$
\left\{\begin{array}{l}
\gamma^{\prime}(t)=x_{0} \cdot(\exp t \mathrm{~W}) \text { for } t \geqq 0 \\
\mathrm{~W}=\left(d \rho_{e}\right)^{-1}\left(\frac{1}{2 \sqrt{n l}} \operatorname{diag}(-1, \underbrace{0, \ldots, 0}_{n-3}, 1,0, \ldots\right. \\
\ldots,-1, \underbrace{0, \ldots, 0}_{n-3}, 1,0)) .
\end{array}\right.
$$

So, from now on to the paragraph including the formula (5.4), we suppose $n \geqq 3$. And we "change" the direction of $x_{0} \cdot H_{\Theta} N_{\Theta}$ in the horosphere $\mathcal{L}=x_{0} \cdot M_{\Theta} N_{\Theta}$ so that it projects onto a compact subset of $X / \Gamma^{\prime}$.

First, we recall that $H_{\Theta}=\bar{M} \bar{A} A^{\perp}(\S 3)$.

Let $\mathbf{k}^{\prime}$ be a totally real algebraic number field of degree $n-1$ over $\mathbf{Q}$ such that $\mathbf{k} \cap \mathbf{k}^{\prime}=\mathbf{Q}$. We can take such an algebraic number field $\mathbf{k}^{\prime}$ as follows. (The author learned it from Dr. C. Nakayama.) Let $p$ be a prime such that $p \equiv 1 \bmod 2(n-1)$ and that $p$ is unramified in $\mathbf{k} \mid \mathbf{Q}$. Let $\zeta_{p}$ be a primitive $p$ th root of unity. Then the field $\mathbf{Q}\left(\zeta_{p}+\zeta_{p}^{-1}\right)$ is a Galois extension of $\mathbf{Q}$ and totally real. Since the Galois group of $\mathbf{Q}\left(\zeta_{p}+\zeta_{p}^{-1}\right)$ over $\mathbf{Q}$ is the cyclic group of order $(p-1) / 2$, we can take a normal subgroup of index $n-1$. Take the corresponding intermediate field. Then this field is the desired field $\mathbf{k}^{\prime}$.

Let $\left\{\alpha_{1}^{\prime}, \ldots, \alpha_{n-1}^{\prime}\right\}$ be an integral basis of $\mathbf{k}^{\prime}$ and

$$
\alpha_{i}^{\prime} \alpha_{j}^{\prime}=\sum_{s=1}^{n-1}{b^{\prime}}_{i j}^{s} \alpha_{s}^{\prime} ; b_{i j}^{\prime s} \in \mathbf{Z}
$$


for $i, j \in\{1, \ldots, n-1\}$. For each $k=1, \ldots, n-1$, let $\mathbf{B}_{k}^{\prime}=\left(b_{k i}^{j}\right)$, where the $i$ - $j$ element of $\mathbf{B}_{k}^{\prime}$ is $b_{k i}^{\prime j}$. For $Z=\left(z_{i j}\right) \in \mathfrak{g l}(n-1, \mathbf{C})$, we consider the following system of linear equations:

$\left(\begin{array}{llll}z_{i 1} & z_{i 2} & \ldots & z_{i, n-1}\end{array}\right)=\left(\begin{array}{llll}z_{11} & z_{12} & \ldots & z_{1, n-1}\end{array}\right) \mathbf{B}_{1}^{\prime-1} \mathbf{B}_{i}^{\prime} \quad ; i=2, \ldots n-1$.

Let $\mathbf{H}(\subset \mathfrak{g l}(n-1, \mathbf{C}))$ be the algebraic group defined by the system of equations (5.2) and the condition $\operatorname{det} Z=1$. Let $\phi_{1}^{\prime}=i d ., \phi_{2}^{\prime}, \ldots, \phi_{n-1}^{\prime}$ : $\mathbf{k}^{\prime} \longrightarrow \mathbf{C}$ be the distinct field embeddings, and $\mathbf{S}^{\prime}=\left(\phi_{j}^{\prime}\left(\alpha_{i}^{\prime}\right)\right)$ be the $(n-$ 1) $\times(n-1)$ matrix. We define a representation $\rho^{\prime}: \mathbf{H} \longrightarrow \mathfrak{g l}(n-1, \mathbf{C})$ by $\rho^{\prime}(Z)=\mathbf{S}^{\prime-1} Z \mathbf{S}^{\prime}$ for $Z \in \mathbf{H}$. We also define an embedding $\iota^{\prime}: \mathbf{k}^{\prime} \longrightarrow$ $\mathfrak{g l}(n-1, \mathbf{C})$ by $\iota^{\prime}\left(\alpha^{\prime}\right)=\operatorname{diag}\left(\phi_{1}^{\prime}\left(\alpha^{\prime}\right), \phi_{2}^{\prime}\left(\alpha^{\prime}\right), \ldots, \phi_{n-1}^{\prime}\left(\alpha^{\prime}\right)\right)$ for $\alpha^{\prime} \in \mathbf{k}^{\prime}$. Then the group $\mathbf{H}$ is defined over $\mathbf{Q}$, and $H_{\mathbf{Z}}$ is isomorphic to the group $U^{\prime}$ of all units in the ring $\mathcal{O}_{\mathbf{k}^{\prime}}$ of algebraic integers of $\mathbf{k}^{\prime}$ under the map $\iota^{\prime-1} \circ \rho^{\prime}$. (The group $\mathbf{H}_{\mathbf{Z}}$ is essentially the same as the transpose of the group $K$ constructed in the proof of Proposition 10.4.10 of [5, p.237].) We remark that $\rho^{\prime}\left(\mathbf{H}_{\mathbf{Z}} \cap\left(\mathbf{H}_{\mathbf{R}}\right)^{0}\right)$ is a subgroup of finite index of $\iota^{\prime}\left(U^{\prime}\right)$. Therefore, by the Dirichlet units theorem, $\mathbf{H}_{\mathbf{R}} / \mathbf{H}_{\mathbf{Z}}$ is compact. Let

$$
\widetilde{\mathbf{H}}=\left\{\left(\begin{array}{ll}
Z & \\
& 1
\end{array}\right) \mid Z \in \mathbf{H}\right\} \subset S L(n, \mathbf{C}) .
$$

Then $\widetilde{\mathbf{H}}$ is an algebraic Q-subgroup of $S L(n, \mathbf{C})$. And the quotient $\widetilde{\mathbf{H}}_{\mathbf{R}} / \widetilde{\mathbf{H}}_{\mathbf{Z}}$ is compact. By Borel-Harish-Chandra's theorem ([4, Theorem 12.3], we have $X_{\mathbf{Q}}(\widetilde{\mathbf{H}})=1$, where $X_{\mathbf{Q}}(\widetilde{\mathbf{H}})$ is the group of $\mathbf{Q}$-characters of $\widetilde{\mathbf{H}}$. By the construction of $\widetilde{\mathbf{H}}$, we have $X_{\mathbf{k}^{\prime}}(\widetilde{\mathbf{H}})=X(\widetilde{\mathbf{H}}) \cong \mathbf{Z}^{n-2}$, where $X_{\mathbf{k}^{\prime}}(\widetilde{\mathbf{H}})$ (resp. $X(\widetilde{\mathbf{H}})$ ) is the group of $\mathbf{k}^{\prime}$-characters (resp. characters) of $\widetilde{\mathbf{H}}$. Let $\hat{\mathbf{H}}=\rho^{-1} \circ \iota(\tilde{\mathbf{H}})$ be the group obtained from $\widetilde{\mathbf{H}}$ by the restriction of scalars from $\mathbf{k}$ to $\mathbf{Q}$ as in $\S 1$. Since $\mathbf{k} \cap \mathbf{k}^{\prime}=\mathbf{Q}$, the group $X_{\mathbf{k}}(\widetilde{\mathbf{H}})$ of $\mathbf{k}$-characters of $\widetilde{\mathbf{H}}$ is trivial. Therefore, the quotient $\hat{\mathbf{H}}_{\mathbf{R}} / \hat{\mathbf{H}}_{\mathbf{Z}}$ is compact by the BorelHarish-Chandra's theorem.

We put $\mathfrak{d}=\left(\operatorname{det} \mathbf{S}^{\prime}\right)^{n-1} \mathbf{S}^{\prime-1}$ if $\operatorname{det} \mathbf{S}^{\prime}>0$,

$$
\mathfrak{d}=\left(-\operatorname{det} \mathbf{S}^{\prime}\right)^{n-1} \operatorname{diag}(-1, \underbrace{1, \ldots, 1}_{n-1}) \mathbf{S}^{\prime-1}
$$

if $\operatorname{det} \mathbf{S}^{\prime}<0$. Then, $\mathfrak{d} \in S L(n-1, \mathbf{R})$. Let $R=\rho^{-1}(\operatorname{diag}(\mathfrak{d}, 1, \ldots, \mathfrak{d}, 1)) \in$ $M_{\Theta}$. We have $\bar{M} \bar{A}=R\left(\hat{\mathbf{H}}_{\mathbf{R}}\right)^{0} R^{-1}, R^{-1} M_{\Theta} R=M_{\Theta}$, and $R^{-1} N_{\Theta} R=N_{\Theta}$. Each element of $A^{\perp}$ commutes with $R$. We put $\Lambda=\hat{\mathbf{H}}_{\mathbf{Z}} \subset \Gamma=\mathbf{G}_{\mathbf{Z}}$. Then the quotient $x_{0} \cdot R^{-1} \bar{M} \bar{A} R / \Lambda$ is compact. Since $\left[\Gamma: \Gamma^{\prime}\right]<\infty$, we 
can assume that $\Lambda \subset \Gamma^{\prime}$ by replacing it with its subgroup of finite index if necessary.

According to the Dirichlet units theorem, the group $U$ of all units in $\mathcal{O}_{\mathbf{k}}$ is isomorphic to the direct product $W \times \mathbf{Z}^{l-1}$, where $W$ is the finite cyclic group of even order consisting of all roots of unity in $U$. We take a set $\left\{v_{1}, \ldots, v_{l-1}\right\} \subset U$ of generators of $\mathbf{Z}^{l-1}$ under the above isomorphism. For each $i \in\{1, \ldots, l-1\}$, let $\sigma_{1, i}$ be the element of $\Gamma \cap A^{\perp}$ given by

$$
\left\{\begin{array}{l}
\sigma_{1, i}=\rho^{-1}\left(\operatorname{diag}\left(\mathfrak{m}_{i}^{1}, \ldots, \mathfrak{m}_{i}^{l}\right)\right), \\
\mathfrak{m}_{i}^{k}=\operatorname{diag}\left(\phi_{k}\left(v_{i}\right)^{-1}, \ldots, \phi_{k}\left(v_{i}\right)^{-1}, \phi_{k}\left(v_{i}\right)^{n-1}\right) ; k=1, \ldots l,
\end{array}\right.
$$

where each $\mathfrak{m}_{i}^{k}$ is an $n \times n$ matrix such that its $n$-n entry is $\phi_{1}\left(v_{i}\right)^{n-1}$, and other diagonal elements are all equal to $\phi_{1}\left(v_{i}\right)^{-1}$.

Recall that $\left\{\alpha_{1}, \ldots, \alpha_{l}\right\}$ is an integral basis of $\mathbf{k}$.

For each $i \in\{1, \ldots, n-1\}$ and $j \in\{1, \ldots, l\}$, let $\sigma_{2, i, j}$ be the element of $\Gamma \cap N_{\Theta}$ given by

$$
\left\{\begin{array}{l}
\sigma_{2, i, j}=\rho^{-1}\left(\left(\begin{array}{ccccc}
I_{n-1} & { }^{t}\left(\xi_{i, j}^{1}\right) & & & \\
& 1 & & & \\
& & \ddots & & \\
& & & I_{n-1} & { }^{t}\left(\xi_{i, j}^{l}\right) \\
& & & 1
\end{array}\right)\right. \\
\xi_{i, j}^{k}=\left(0, \ldots, 0, \phi_{k}\left(\alpha_{j}\right), 0 \ldots, 0\right): k=1, \ldots, l,
\end{array}\right.
$$

where the $i$-th entry of $\xi_{i, j}^{k}$ is $\phi_{k}\left(\alpha_{j}\right)$ and other entries are all equal to zero. Since $\left[\Gamma: \Gamma^{\prime}\right]<\infty$, we can assume that $\sigma_{1, i}, \sigma_{2, i, j} \in \Gamma^{\prime}$ for all $i, j$ by replacing them with their powers if necessary. We denote by $\Lambda^{\prime}$ the subgroup of $\Gamma^{\prime}$ generated by

$$
\Lambda \cup\left\{\sigma_{1, i} \mid i=1, \ldots, l-1\right\} \cup\left\{\begin{array}{l|l}
\sigma_{2, i, j} & \begin{array}{l}
i=1, \ldots, n-1 \\
j=1, \ldots, l
\end{array}
\end{array}\right\} .
$$

Then $R^{-1} H_{\Theta} N_{\Theta} R / \Lambda^{\prime}$ is compact, and hence $\Pi\left(x_{0} \cdot R^{-1} H_{\Theta} N_{\Theta} R\right)$ is compact.

In order to simplify the succeeding description, we also put $R=I_{2 l}$ in the case $n=2$.

We define a diffeomorphism $\Xi: x_{0} \cdot H_{\Theta} N_{\Theta} \longrightarrow x_{0} \cdot R^{-1} H_{\Theta} N_{\Theta} R$ by $\Xi\left(x_{0} \cdot g_{1} g_{2}\right)=x_{0} \cdot R^{-1} g_{1} g_{2} R$ for $g_{1} \in H_{\Theta}, g_{2} \in N_{\Theta}$. We remark that both $H_{\Theta} N_{\Theta}$ and $R^{-1} H_{\Theta} N_{\Theta} R$ have group structures. The map $\Xi$ is a bilipschitz diffeomorphism between the Riemannian manifolds $x_{0} \cdot H_{\Theta} N_{\Theta}$ and $x_{0} \cdot R^{-1} H_{\Theta} N_{\Theta} R$. 
Now we define a new $\{(n-1) l-1\}$-cycle $\mathcal{Z}_{m}$ (resp. $(n-1) l$-chain $\left.\mathcal{U}_{m}\right)$ by $\mathcal{Z}_{m}=\Xi \circ z_{m}\left(\right.$ resp. $\left.\mathcal{U}_{m}=\Xi \circ u_{m}\right)$ for each positive integer $m$.

Lemma 5.5. The pullback of the restriction of $\Omega$ to $x_{0} \cdot R^{-1} H_{\Theta} N_{\Theta} R$ under the map $\Xi$ is identical with the restriction of $\Omega$ to $x_{0} \cdot H_{\Theta} N_{\Theta}$ : i.e. $\Xi^{*}\left(\left.\Omega\right|_{x_{0} \cdot R^{-1} H_{\Theta} N_{\Theta} R}\right)=\left.\Omega\right|_{x_{0} \cdot H_{\Theta} N_{\Theta}}$.

Proof. Let $n_{\mathbf{y}}$ for $\mathbf{y}=\left(y_{1}, \ldots, y_{(n-1) l}\right) \in \mathbf{R}^{(n-1) l}$ as in the proof of Lemma 4.2.

We define a linear map $\widehat{R}: \mathbf{R}^{(n-1) l} \longrightarrow \mathbf{R}^{(n-1) l}$ by

$$
{ }^{t}(\widehat{R} \mathbf{y})=\left(\begin{array}{lll}
\mathfrak{d}^{-1} & & \\
& \ddots & \\
& & \mathfrak{d}^{-1}
\end{array}\right){ }^{t} \mathbf{y} \quad \text { for } \mathbf{y} \in \mathbf{R}^{(n-1) l} .
$$

Suppose that

$$
\left\{\begin{array}{l}
g=g_{1} g_{2} \in H_{\Theta} N_{\Theta} \\
g_{1}=\rho^{-1}\left(\operatorname{diag}\left(B_{1}, b_{1}, \ldots, B_{l}, b_{l}\right)\right) \in H_{\Theta} \\
g_{2}=n_{\mathbf{y}} \in N_{\Theta}
\end{array}\right.
$$

where each $B_{k}$ is an $(n-1) \times(n-1)$ diagonal matrix. Then we have

$$
R^{-1} g R=\rho^{-1}\left(\operatorname{diag}\left(\mathfrak{d}^{-1} B_{1} \mathfrak{d}, b_{1}, \ldots, \mathfrak{d}^{-1} B_{l} \mathfrak{d}, b_{l}\right)\right) n_{\widehat{R} \mathbf{y}}
$$

and $\mathcal{P}_{f}\left(x_{0} \cdot R^{-1} g R\right)=\widehat{R} \mathbf{y}$. So, $\mathcal{P}_{f} \circ \Xi=\widehat{R} \circ \mathcal{P}_{f}$.

Since $\operatorname{det}\left(\begin{array}{lll}\mathfrak{d}^{-1} & & \\ & \ddots & \\ & & \mathfrak{d}^{-1}\end{array}\right)=1$, we obtain

$$
\begin{aligned}
\Xi^{*}\left(\left.\Omega\right|_{x_{0} \cdot R^{-1} H_{\Theta} N_{\Theta} R}\right) & =\Xi^{*} \circ \mathcal{P}_{f}^{*}\left(\left.\Omega\right|_{x_{0} \cdot N_{\Theta}}\right)=\mathcal{P}_{f}^{*} \circ \widehat{R}^{*}\left(\left.\Omega\right|_{x_{0} \cdot N_{\Theta}}\right) \\
& =\mathcal{P}_{f}^{*}\left(\left.\Omega\right|_{x_{0} \cdot N_{\Theta}}\right)=\left.\Omega\right|_{x_{0} \cdot H_{\Theta} N_{\Theta}}
\end{aligned}
$$

Definition 5.6. We define the injectivity radius $\operatorname{Injrad}_{x}$ at $x \in X$ to be the injectivity radius at $\Pi(x)$ on the Riemannian manifold $X / \Gamma^{\prime}$ : i.e.

$$
\operatorname{Injrad}_{x}=\frac{1}{2} \min \left\{d_{X}(x, x \cdot \sigma) \mid \sigma \in \Gamma^{\prime}, \sigma \neq i d .\right\} .
$$


Lemma 5.7. There exists a positive number $C$ such that the following holds for each $t \in \mathbf{R}$ :

$$
\operatorname{Injrad}_{x} \leqq C e^{\frac{-t}{\sqrt{2(n-1) l}}}
$$

for any point $x$ on the horosphere $h_{\gamma}^{-1}(-t)$.

Proof. Let $\overline{\mathbf{G}}=\rho^{-1}\left((S L(n-1, \mathbf{C}))^{l}\right), \overline{\mathbf{P}}$ be as in $\S 3$. We put $\overline{\Gamma^{\prime}}=\Gamma^{\prime} \cap \overline{\mathbf{G}}$. Since $\overline{\Gamma^{\prime}}$ is an arithmetic subgroup of $\overline{\mathbf{G}}$, the double coset classes $\overline{\mathbf{P}}_{\mathbf{Q}} \backslash \overline{\mathbf{G}}_{\mathbf{Q}} /{\overline{\Gamma^{\prime}}}^{\prime}$ are known to be finite by Borel [3]. Let $\bar{\sigma}_{1}, \ldots, \bar{\sigma}_{\nu}$ be a complete representative system of $\overline{\mathbf{P}}_{\mathbf{Q}} \backslash \overline{\mathbf{G}}_{\mathbf{Q}} / \overline{\Gamma^{\prime}}$. We assume the following.

$$
\left\{\begin{array}{l}
\rho\left(\bar{\sigma}_{i}\right)=\operatorname{diag}\left(\phi_{1}\left(\mathfrak{c}_{i}\right), 1, \ldots, \phi_{l}\left(\mathfrak{c}_{i}\right), 1\right), \\
\mathfrak{c}_{i} \in S L(n-1, \mathbf{k}) \quad \text { for } i=1, \ldots, \nu .
\end{array}\right.
$$

We remark that the horosphere

$$
h_{\gamma}^{-1}(-t)=x_{0} \cdot M_{\Theta} a_{t} N_{\Theta}=x_{0} \cdot \bar{M} \bar{A} \bar{N} A^{\perp} a_{t} N_{\Theta}
$$

is invariant under the right translation by each element of $M_{\Theta}$.

By virtue of the Borel's result ([3]) on the fundamental open set for $\overline{\Gamma^{\prime}}$ combined with the use of the action of the group generated by

$$
\left\{\sigma_{1, i} \mid i=1, \ldots, l-1\right\}
$$

we can assume the following.

(5.7.2) There exist positive constants $C_{1}, C_{2}, C_{3}, C_{4}$ such that the following holds. For any $x \in h_{\gamma}^{-1}(-t)$, there exist $\mu \in\{1, \ldots, \nu\}$ and $x^{\prime} \in h_{\gamma}^{-1}(-t)$ of the following form such that $\Pi(x)=\Pi\left(x^{\prime}\right)$ :

$$
x^{\prime}=x_{0} \cdot g_{2} g_{3} \bar{\sigma}_{\mu} g_{4} a_{t} n_{\mathbf{y}},
$$

$$
\begin{aligned}
& g_{2}=\rho^{-1}\left(\operatorname{diag}\left(\beta_{1}^{1}, \ldots, \beta_{n-1}^{1}, 1, \ldots \ldots, \beta_{1}^{l}, \ldots, \beta_{n-1}^{l}, 1\right)\right) \\
& \quad \times \rho^{-1}\left(\operatorname{diag}\left(\kappa_{1}, \ldots, \kappa_{n-1}, 1, \ldots \ldots, \kappa_{1}, \ldots, \kappa_{n-1}, 1\right)\right) \in \bar{M} \bar{A} \\
& \beta_{i}^{k}>0 \text { for all } i, k ; \prod_{i=1}^{n-1} \beta_{i}^{k}=1 \text { for all } k ; \prod_{k=1}^{l} \beta_{i}^{k}=1 \text { for all } i \\
& \kappa_{i}>0 \text { for all } i ; \kappa_{1} \cdots \cdots \kappa_{n-1}=1,
\end{aligned}
$$


(5.7.2.c)

$$
\begin{aligned}
& g_{3}=\rho^{-1}\left(\operatorname{diag}\left(\mathfrak{n}_{1}, 1, \ldots, \mathfrak{n}_{l}, 1\right)\right) \in \bar{N} \\
& \text { where each } \mathfrak{n}_{k}=\left(\begin{array}{ccc}
1 & & b_{i, j}^{k} \\
& \ddots & \\
& & 1
\end{array}\right) \text { is an upper triangular, real }(n-1)
\end{aligned}
$$

$\times(n-1)$ matrix such that its diagonal entries are all equal to 1 ,

$$
g_{4}=\rho^{-1}\left(\operatorname{diag}\left(q_{1}, \ldots, q_{1},\left(q_{1}\right)^{1-n}, \ldots \ldots, q_{l}, \ldots, q_{l},\left(q_{l}\right)^{1-n}\right)\right) \in A^{\perp}
$$

$$
\begin{gathered}
\frac{\kappa_{i}}{\kappa_{i+1}} \leqq C_{1} \quad \text { for all } i \\
\frac{1}{C_{2}} \leqq \beta_{i}^{k} \leqq C_{2} \quad \text { for all } i, k \\
\left|b_{i, j}^{k}\right| \leqq C_{3} \quad \text { for all } i, j, k \\
\frac{1}{C_{4}} \leqq q_{k} \leqq C_{4} \quad \text { for all } k
\end{gathered}
$$

For each $i$, we can find an element $\mathfrak{v}_{i}$ of $\mathbf{k}^{n-1}$ such that $\mathfrak{c}_{i}\left({ }_{\mathfrak{v}_{i}}\right)=$ ${ }^{t}(1,0, \ldots, 0)$, because $\mathfrak{c}_{i} \in S L(n-1, \mathbf{k})$. By replacing $\mathfrak{v}_{1}, \ldots, \mathfrak{v}_{\nu}$ with their constant multiples if necessary, we can assume the following.

There exist positive integer $C_{5}$ and elements $\mathfrak{v}_{1}, \ldots, \mathfrak{v}_{\nu}$ of

$$
\left(\mathcal{O}_{\mathbf{k}}\right)^{n-1} \text { such that } \mathfrak{c}_{i}\left({ }_{\mathfrak{v}_{i}}\right)={ }^{t}\left(C_{5}, 0, \ldots, 0\right) \text { for } i=1, \ldots, \nu \text {. }
$$

Let $\mathbf{y}_{j}=\left(\phi_{1}\left(\mathfrak{v}_{j}\right), \ldots, \phi_{l}\left(\mathfrak{v}_{j}\right)\right) ; j=1, \ldots, \nu$, where each $\phi_{i}: \mathbf{k}^{n-1} \longrightarrow \mathbf{C}^{n-1}$ is the natural linear map induced from $\phi_{i}$. By replacing $n_{\mathbf{y}_{1}}, \ldots, n_{\mathbf{y}_{\nu}}$ with their powers if necessary, we can assume that

$$
n_{\mathbf{y}_{1}}, \ldots, n_{\mathbf{y}_{\nu}} \in \Gamma^{\prime}
$$

Let $g_{2} g_{3}=\rho^{-1}\left(\operatorname{diag}\left(B_{1}, 1, \ldots, B_{l}, 1\right)\right)$ and $x^{\prime}=x_{0} \cdot n_{\mathbf{y}^{\prime}} g_{2} g_{3} \bar{\sigma}_{\mu} g_{4} a_{t}$. Then we have

$$
{ }^{t} \mathbf{y}^{\prime}=e^{-\frac{t}{\sqrt{2(n-1) l}}}\left(\begin{array}{lll}
\left(q_{1}\right)^{n} B_{1} & & \\
& \ddots & \\
& & \left(q_{l}\right)^{n} B_{l}
\end{array}\right)\left(\begin{array}{ccc}
\phi_{1}\left(\mathfrak{c}_{\mu}\right) & & \\
& \ddots & \\
& & \phi_{l}\left(\mathfrak{c}_{\mu}\right)
\end{array}\right){ }^{t} \mathbf{y}
$$


where each $\phi_{i}: S L(n-1, \mathbf{k}) \longrightarrow S L(n-1, \mathbf{R})$ is the natural homomorphism induced from $\phi_{i}$. So, if $x^{\prime} \cdot n_{\mathrm{y}_{\mu}}=x_{0} \cdot n_{\mathrm{y}^{\prime \prime}} g_{2} g_{3} \bar{\sigma}_{\mu} g_{4} a_{t}$, then we have

$$
\begin{aligned}
& { }^{t}\left(\mathbf{y}^{\prime \prime}-\mathbf{y}^{\prime}\right) \\
& =e^{-\frac{t}{\sqrt{2(n-1) l}}}\left(\begin{array}{lll}
\left(q_{1}\right)^{n} B_{1} & & \\
& \ddots & \\
& & \left(q_{l}\right)^{n} B_{l}
\end{array}\right)\left(\begin{array}{lll}
\phi_{1}\left(\mathfrak{c}_{\mu}\right) & & \\
& \ddots & \\
& & \phi_{l}\left(\mathfrak{c}_{\mu}\right)
\end{array}\right){ }^{t} \mathbf{y}_{\mu} \\
& =e^{-\frac{t}{\sqrt{2(n-1) l}}} \operatorname{diag}\left(\left(q_{1}\right)^{n} B_{1}, \ldots,\left(q_{l}\right)^{n} B_{l}\right) \\
& \times{ }^{t}\left(C_{5}, 0, \ldots, 0, \ldots \ldots, C_{5}, 0, \ldots, 0\right) \\
& =C_{5} e^{-\frac{t}{\sqrt{2(n-1) l}} t}\left(\left(q_{1}\right)^{n} \kappa_{1} \beta_{1}^{1}, 0, \ldots, 0, \ldots \ldots,\left(q_{l}\right)^{n} \kappa_{1} \beta_{1}^{l}, 0, \ldots, 0\right) .
\end{aligned}
$$

Hence,

$$
\begin{aligned}
\text { Injrad }_{x} & =\operatorname{Injrad}_{x^{\prime}} \\
& \leqq \frac{1}{2} d_{X}\left(x^{\prime}, x^{\prime} \cdot n_{\mathbf{y}_{\mu}}\right)=\frac{1}{2} d_{X}\left(x_{0} \cdot n_{\mathbf{y}^{\prime}}, x_{0} \cdot n_{\mathbf{y}^{\prime \prime}}\right) \\
& \leqq \frac{1}{2} C_{5} e^{-\frac{t}{\sqrt{2(n-1) !}}} \kappa_{1} \sqrt{n \times\left\{\left(q_{1}\right)^{2 n}\left(\beta_{1}^{1}\right)^{2}+\cdots+\left(q_{l}\right)^{2 n}\left(\beta_{1}^{l}\right)^{2}\right\}} .
\end{aligned}
$$

From (5.7.2.e) and that $\kappa_{1} \cdots \cdots \kappa_{n-1}=1$, we have $\kappa_{1} \leqq\left(C_{1}\right)^{n / 2-1}$. Thus (5.7.2.f) and (5.7.2.h) imply that

$$
\begin{aligned}
\text { Injrad }_{x} & \leqq \frac{1}{2} C_{5} e^{-\frac{t}{\sqrt{2(n-1) l}}}\left(C_{1}\right)^{n / 2-1} \sqrt{n \times\left\{\left(C_{4}\right)^{2 n}\left(C_{2}\right)^{2} \times l\right\}} \\
& =\frac{1}{2}\left(C_{1}\right)^{n / 2-1} C_{2}\left(C_{4}\right)^{n} C_{5} \sqrt{n l} e^{-\frac{t}{\sqrt{2(n-1) l}}} .
\end{aligned}
$$

Therefore, it suffices to put $C=\frac{1}{2}\left(C_{1}\right)^{n / 2-1} C_{2}\left(C_{4}\right)^{n} C_{5} \sqrt{n l}$.

Concerning the function $f: X / \Gamma^{\prime} \longrightarrow \mathbf{R}$ in $\S 1$, we have

$$
\Pi\left(x_{0} \cdot R^{-1} H_{\Theta} N_{\Theta} R\right) \subset f^{-1}\left(\left[t_{0}, t_{1}\right]\right)
$$

for a sufficiently large $t_{1}>0$, because $\Pi\left(x_{0} \cdot R^{-1} H_{\Theta} N_{\Theta} R\right)$ is compact. Let $X^{\prime}=\widetilde{f}^{-1}\left(\left[t_{0}, t_{1}\right]\right)$ and $\varepsilon_{1}>0$ be the minimum of the injectivity radii on $X^{\prime}$. From Lemma 5.7, we have $X^{\prime} \cap h_{\gamma}^{-1}\left(\left(-\infty,-t_{2}\right)\right)=\phi$ for a sufficiently large $t_{2}>0$. Hence it suffices to consider the isoperimetric inequalities on the complement of the horoball $h_{\gamma}^{-1}\left(\left(-\infty,-t_{2}\right)\right)$.

Lemma 5.8. For any lipschitz $(n-1) l$-chain $\mathcal{U}_{m}^{\prime}$ in $X-h_{\gamma}^{-1}\left(\left(-\infty,-t_{2}\right)\right)$ which bounds $\mathcal{Z}_{m}$, we have

$$
\operatorname{mass}_{(n-1) l}\left(\mathcal{U}_{m}^{\prime}\right) \geqq 2^{(n-1) l} n^{(n-1) l / 2} e^{-\sqrt{(n-1) l / 2} t_{2}} \cdot e^{n(n-1) l \cdot m} .
$$


Proof. Since $\mathrm{H}^{(n-1) l}\left(X-h_{\gamma}^{-1}\left(\left(-\infty,-t_{2}\right)\right)\right)=0$, we can take an $\{(n-1) l-1\}$ -form $\omega$ such that $\Omega=d \omega$. Lemma 5.5 implies that

$$
\begin{aligned}
\int_{\mathcal{U}^{\prime} m} \Omega & =\int_{\mathcal{Z}_{m}} \omega=\int_{\mathcal{U}_{m}} \Omega=\int_{\Xi o u_{m}} \Omega=\int_{u_{m}} \Xi^{*} \Omega=\int_{u_{m}} \Omega \\
& =n^{(n-1) l / 2}\left(2 e^{n m}\right)^{(n-1) l}=2^{(n-1) l} n^{(n-1) l / 2} e^{n(n-1) l \cdot m} .
\end{aligned}
$$

From Lemma 4.2, we have

$$
\|\Omega\|_{x} \leqq e^{\sqrt{(n-1) l / 2} t_{2}} \quad \text { for all } x \in X-h_{\gamma}^{-1}\left(\left(-\infty,-t_{2}\right)\right) .
$$

Hence,

$$
\begin{aligned}
\operatorname{mass}_{(n-1) l}\left(\mathcal{U}_{m}^{\prime}\right) & \geqq e^{-\sqrt{(n-1) l / 2} t_{2}} \int_{\mathcal{U}^{\prime}{ }_{m}} \Omega \\
& =2^{(n-1) l} n^{(n-1) l / 2} e^{-\sqrt{(n-1) l / 2} t_{2}} \cdot e^{n(n-1) l \cdot m} .
\end{aligned}
$$

We are now ready to prove the theorem.

Proof of Theorem. We know by Corollary 3.2 that $\operatorname{mass}_{(n-1) l}\left(\mathcal{Z}_{m}\right)$ and $\operatorname{diam}\left(\mathcal{Z}_{m}\right)$ are bounded above by some polynomials in $m$, because $\Xi$ is a bilipschitz diffeomorphism. This and Lemma $5.8 \mathrm{imply}$ that the isoperimetric inequality does not hold on $X^{\prime}$. Therefore, $\Gamma=S L\left(n, \mathcal{O}_{\mathbf{k}}\right)$ is not combable.

Q.E.D.

Remark. The isoperimetric inequality for $\mathcal{Z}_{m}$ 's does hold on the whole space $X$ : Move $\mathcal{Z}_{m}$ along the geodesics orthogonal to the horosphere $\mathcal{L}$ and fill the resulting cycle $\mathcal{Z}_{m}^{\prime}$. The locus of the cycle $\mathcal{Z}_{m}$ and the chain filling $\mathcal{Z}^{\prime}{ }_{m}$ give us the desired chain. In fact, the fundamental groups of compact manifolds of nonpositive curvature are known to be combable (see [8]). But in the above process, we must move $\mathcal{Z}_{m}$ a distance about a constant multiple of $m$ along the orthogonal direction. If $m$ is large, the chain $\mathcal{Z}_{m}$ crosses the horosphere $h_{\gamma}^{-1}\left(-t_{2}\right)$ on the way to $\mathcal{Z}_{m}^{\prime}$. Therefore we cannot make this construction when we remove the horoball $h_{\gamma}^{-1}\left(\left(-\infty,-t_{2}\right)\right)$.

\section{References.}

[1] J.M. Alonso, Combings of groups, in "Algorithms and Classification in Combinatorial Group Theory", editors G. Baumslag and C.F. Miller III, MSRI Publications;23, Springer-Verlag 1992. 
[2] W. Ballmann, M. Gromov and V. Schroeder, Manifolds of Nonpositive Curvature, Progress in Math., Vol. 61, Birkhäuser, Boston Basel Stuttgart 1985.

[3] A. Borel, Introduction aux Groupes Arithmétiques, Hermann, Paris 1969.

[4] A. Borel and Harish-Chandra, Arithmetic subgroups of algebraic groups, Ann. of Math. 75(1962), 485-535.

[5] J.W. Cannon, D.B.A. Epstein, D.F. Holt, S.V.F. Levy, M.S. Paterson and W.P. Thurston, Word Processing in Groups, Jones and Bartlett, Boston 1992.

[6] P. Eberlein, Structure of manifolds of nonpositive curvature, in Global Differential Geometry and Global Analysis (1984), editors D. Ferus et al., Lecture Notes in Math. 1156, Springer-Verlag 1985, 86-153.

[7] P. Eberlein and B. O'Neil, Visibility manifolds, Pac. J. Math. 46(1973), 45109.

[8] S.M. Gersten, Bounded cocycles and combings of groups, Internat. J. Algebra Comput. 2(1992), 307-326.

[9] S.M. Gersten and H.B.Short, Small cancellation theory and automatic groups, Invent. math. 102(1990), 305-334.

[10] Rational subgroups of biautomatic groups, Ann. of Math. 134(1991), 125-158.

[11] E. Ghys and P. de la Harpe, editors, Sur les groupes hyperboliques d'apr̀es Mikhael Gromov, Progress in Math., Vol. 83, Birkhäuser, Boston 1990.

[12] M. Gromov, Hyperbolic groups, in "Essays in Group Theory", editor S.M. Gersten, MSRI Publications;8, Springer-Verlag 1987.

[13] Asymptotic invariants of infinite groups, in Geometric Group Theory, Vol. 2, editors G.A.Niblo and M.A.Roller, London Math. Soc. Lecture Note Ser. 182, Cambridge University Press 1993.

[14] T. Hattori, Collapsing of quotient spaces of $S O(n) \backslash S L(n, \mathbf{R})$ at infinity, J. Math. Soc. Japan 47(1995), 193-225.

[15] F. Hirzeburch, Hilbert modular surfaces, L'Enseign Math. 19(1973), 183-281.

[16] G.D. Mostow, Strong rigidity of locally symmetric spaces, Annals of Math. Studies 78, Princeton University Press, Princeton, New Jersey 1973.

[17] M.S. Raghunathan, A note on quotients of real algebraic groups by arithmetic subgroups, Invent. Math. 4(1968), 318-335.

[18] R.J. Zimmer, Ergodic Theory and Semisimple Groups, Birkhäuser, Boston 1984. 
[19] A. Weil, Adeles and Algebraic groups, Progress in Math., Vol. 23, Birkhäuser, Boston Basel Stuttgart 1982.

Department of Mathematics

TOKYo InSTITUTE OF TECHNOLOGY

OHokayama, Meguro

TOKYO, 152, JAPAN

RECEIVEd July 11Th, 1994. 